\title{
Geological Prospection of Placer Chromium Deposits in the Waropen Regency-Indonesia (New Guinea) Using the Method of Indicator Minerals
}

\author{
Karol Zglinicki ${ }^{1}$, Paweł Kosiński ${ }^{2}$, Adam Piestrzyński ${ }^{3}$ and Krzysztof Szamałek ${ }^{1,2, *}$ \\ 1 Polish Geological Institute-National Research Institute, 4 Rakowiecka St., 00-975 Warsaw, Poland; \\ karol.zglinicki@pgi.gov.pl \\ 2 Faculty of Geology, University of Warsaw, 93 Żwirki i Wigury St., 02-089 Warsaw, Poland; \\ p.kosinski@student.uw.edu.pl \\ 3 Geophysics and Environmental Protection, Faculty of Geology, University of Science and Technology, \\ 30 Mickiewicza St., 30-059 Cracow, Poland; piestrz@geol.agh.edu.pl \\ * Correspondence: krzysztof.szamalek@uw.edu.pl
}

Received: 6 December 2019; Accepted: 20 January 2020; Published: 22 January 2020

\begin{abstract}
Indicator minerals (IM) have been used in the research of gemstone and base metals for over 100 years. IMs are a main source of information about the occurrence of deposits, hydrothermal and weathering changes, as well as bedrock source and type. Since 2013, base metal mineral exploration has taken place on New Guinea Island (the Indonesian part). The analysis of chromian spinels as IM in beach and river sediments led to the discovery of rich chromian-bearing deposits in the Botawa River sediments. The dominant detrital minerals include chromian spinels, olivine, pyroxenes and serpentine. The source of chromian spinels, olivine and pyroxenes are most likely peridotites from the Earth's mantle zone of the ophiolite series, generated under supra-subduction conditions. The terrigenous deposits contain metamorphic minerals, such as staurolites, andalusites, epidotes, amphiboles and fragments of micas, quartz and chlorite schists. Using ICP-MS analysis of the beach sands, the concentration of $\mathrm{Cr}_{2} \mathrm{O}_{3}$ was determined to be at the level of $1.17 \%$, while in the heavy minerals concentrate from the Botawa River sediment, the content of $\mathrm{Cr}_{2} \mathrm{O}_{3}$ amounts to $24.83 \%$. The authors conclude that the west parts of the Van Rees Mountains are the probable parent rocks for chromium-bearing sediments.
\end{abstract}

Keywords: indicator minerals; chromian spinels; geological exploration; New Guinea Island

\section{Introduction}

Indicator minerals (IM) are minerals that provide important information about potential occurrences of deposits [1], weathering and hydrothermal changes, as well as the source and type of parent rock [2,3]. The IM are macroscopically recognizable and characterized by high density and physical resistance, silt and sand size and are transported over great distances [2]. Geologists have been using IM in the mineral exploration of copper ores [4,5], chromium ores [6], as well as platinum [7,8], gold [9] and gemstones $[10,11]$ for a long time. The presence of IM in the alluvial and sea deposits allows recognizing the type of mineral deposits on a regional scale [12].

The gradual depletion of mineral resources entails conducting extensive geological prospection using both the greenfield and brownfield exploration. One of the most promising world regions with the high geopotential is the area of the Malay Archipelago (Indonesia) situated in the south-east edge of the Eurasian Plate. High seismicity (caused by subduction processes) and volcanism are present in this region [13]. The basement of the west part of the Malay Archipelago is represented by the continental crust, while the east part is dominated by the island arc, ophiolite sequences and younger ocean 
basins [13]. The deposit occurrences in Indonesia are strongly connected to subduction phenomena, acidic and middle volcanism in the island arches as well as with ophiolite complexes [14]. On Indonesian islands, chromites occur in two types ore deposits [15] - primary deposits of podiform in ophiolite series (South Kalimantan Province-Tanah Ambungan; South Sulawesi Province-Baru; Papua-Jayapura) and placer deposits (North Maluku-Poso; Central Sulawesi-Bungku).

Chromian spinels provide the only source for metallic chromium used in steelmaking and in metallurgy, as well as chemical and refractory materials industry. Chromium deposits [16] are associated with large, ultramafic stratiform-type layered intrusions Bushveld Complex-South Africa [17,18]; Kemi Intrusion-Finland [19,20]; Burakovsky Intrusion-Russia [21,22] and podiform-type ophiolite sequences: Massif of Bulqiza-Albany [23]; Muğla Ophiolite-Turkey [24,25]; Zambales Ophiolite Complex, Luzon-Philippines [26-28], forming pods and lenses inside the mafic and ultramafic rock complexes. Over 1100 podiform-type deposits have been documented worldwide, comprising almost 58\% of the world's chromium as a metal [29]. Due to the depletion of chromium deposits from the podiform structures, extensive geological prospections are conducted in many regions of the world. In this prospection, the following IM are used: magnesium olivine, chromite, pyroxene (diopside/enstatite), magnetite, serpentine and in the minority uvarovite, chlorite and talc [6,30].

The region with a high mineral potential is the Waropen Regency located in the northern part of New Guinea Island and the adjacent Cendrawasih Bay (Figure 1). The complicated tectonic history of this region (still insufficiently researched) and the confirmed presence of many mineral deposits motivates mining companies to further onshore and offshore exploration on New Guinea Island.

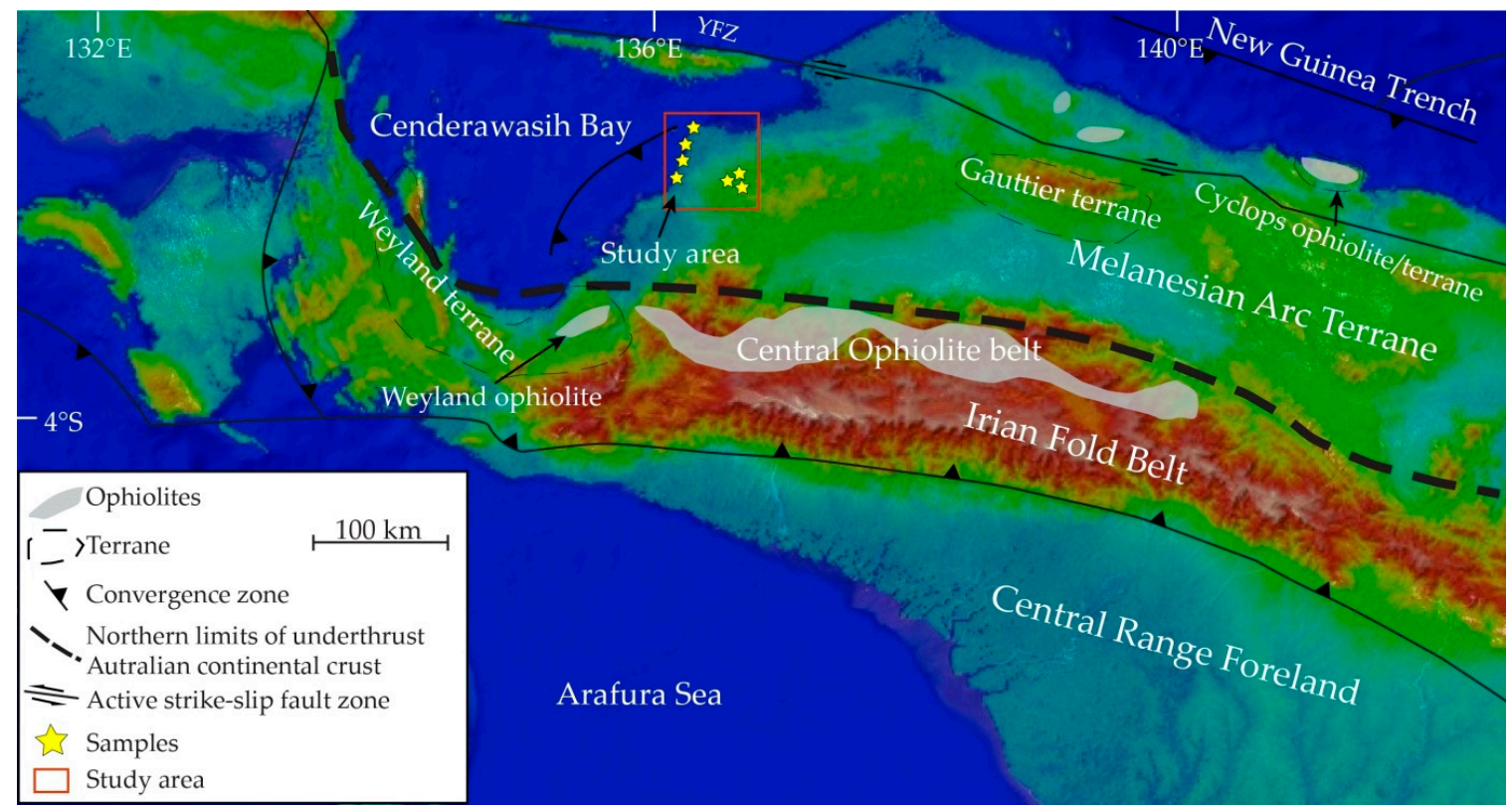

Figure 1. Map of New Guinea Island [31].

Since 2013, a team of Polish geologists [32] has conducted geological prospection in Indonesia and on New Guinea Island. During the geological reconnaissance in the Waropen Regency (Figure 2), samples of beach sediments and alluvial deposits from the Botawa River were collected. Their mineral and chemical composition was determined. Heavy minerals fraction obtained from collected samples consists of characteristic minerals for podiform-type chromium deposits. The rich concentration of chromium spinels indicates the close location of parent rocks. The detailed results of geological prospection in the Waropen Regency are published here for the first time. The main purpose of this research is to identify the rock source and genesis (types of magmas from which this source was formed), as well as the search for chromian spinels placer ore deposits. 


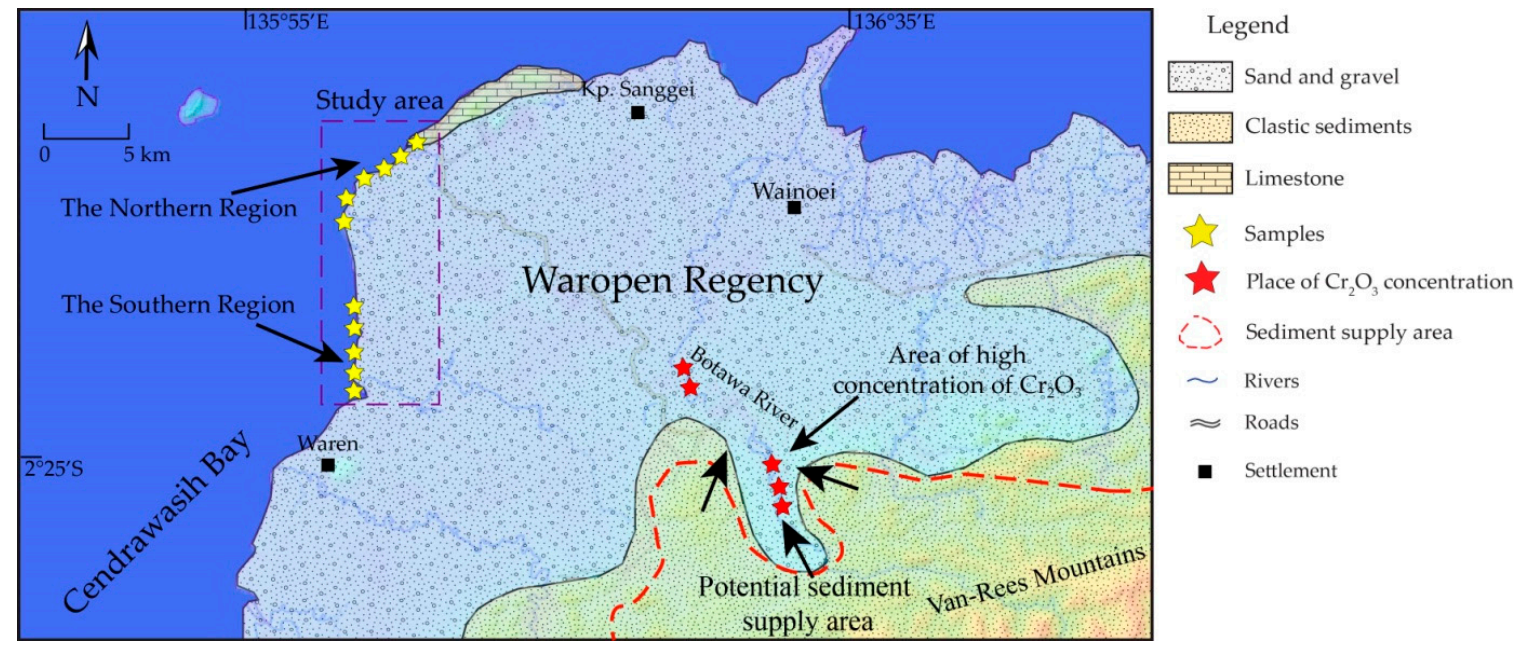

Figure 2. Geology map of the Waropen Regency [33-35].

\section{Materials and Methods}

The geological prospection was carried out along an approximately $8 \mathrm{~km}$ long stretch of the Waropen Regency coastline, between the $136^{\circ} 25^{\prime} 27^{\prime \prime}$ E meridian and $136^{\circ} 21^{\prime} 56^{\prime \prime}$ E meridian. The research was conducted in two regions-North and South. Eleven samples (Figures 2 and 3) of contemporary beach sediments were collected from a depth of 10 to $15 \mathrm{~cm}$ b.g.l. (below ground level).
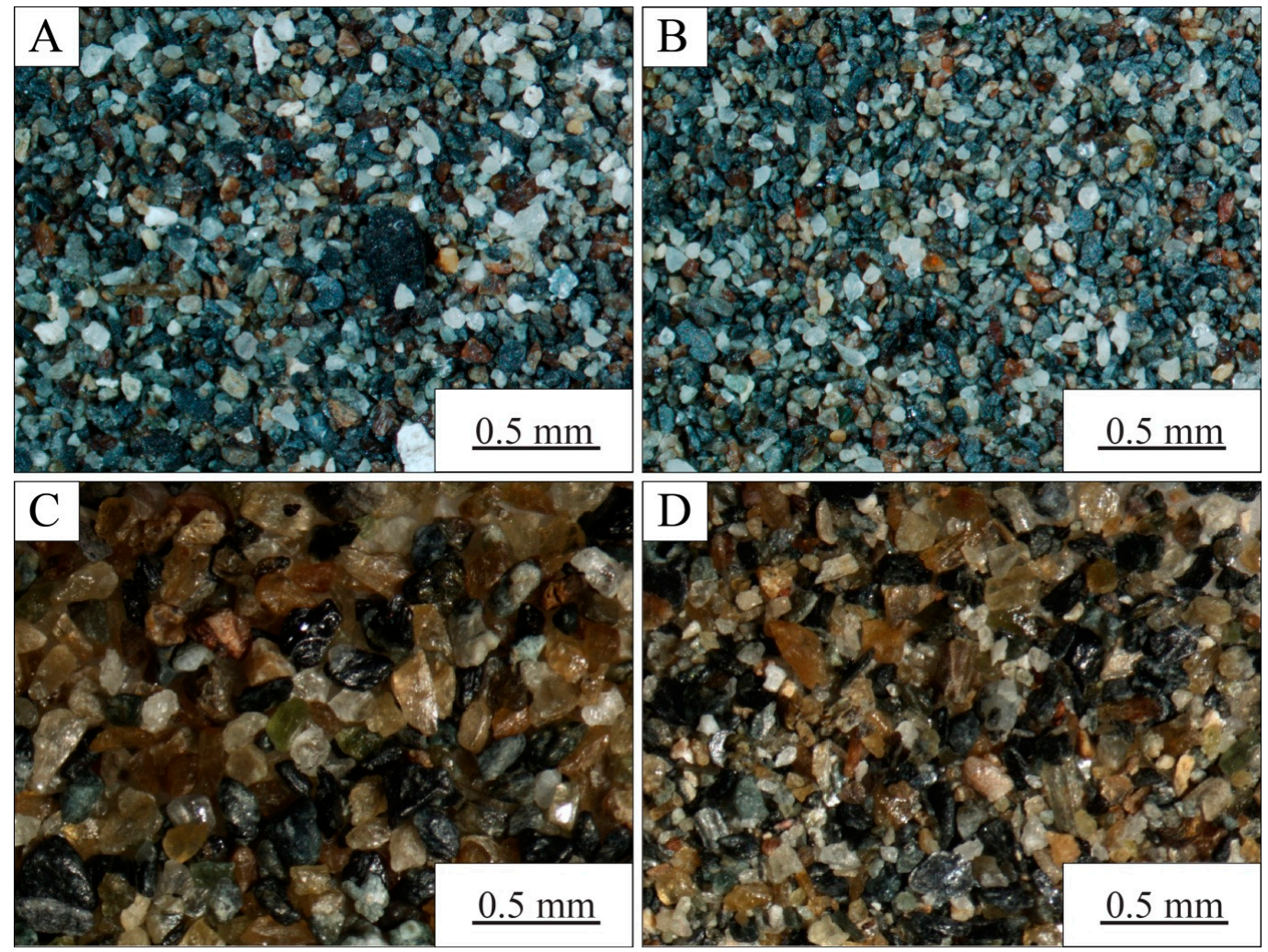

Figure 3. Beach sediments from Waropen Regency. (A) and (B) the southern region samples, dominantly pyroxene and amphibole. Stereoscopic microscope, magnification $\times 2$. (C) and $(\mathbf{D})$ the northern area samples, olivine, pyroxene and chromian spinel grains. Stereoscopic microscope, magnification $\times 4$.

Additionally, 5 pits (red stars, Figure 2) in the alluvial deposit of the Botawa River to a depth of $90 \mathrm{~cm}$ were excavated and 5 samples of heavy minerals concentrate (obtain by panning) were 
collected from there. The weight of extracted samples ranged from 50 to $100 \mathrm{~g}$. Using acetic acid $10 \%$, biogenic elements (shell fragments, foraminifera and snails) were removed. Sieve analysis of the grain material was carried out with sieves with a mesh diameter ranging from $2 \mathrm{~mm}$ to $0.063 \mathrm{~mm}$. Open source GRADISTAT v.5.11 program was used to perform statistical calculations [36]. Statistical parameters of samples were calculated using the Folk\&Ward method [37] ( $\phi$ method). Heavy fraction (from 0.5 to $0.063 \mathrm{~mm}$ ) has been separated from sediments, using sodium polytungstate of $2.89 \mathrm{~g} / \mathrm{cm}^{3}$ density. The heavy minerals' thin sections were embedded in epoxy resin. ECLIPSE E600 POL polarizing microscope and a scanning electron microscope FE-SIGMA VP (Carl Zeiss Microscopy Ltd., Cambridge, UK) with two detectors EDS (SDD XFlash | 10) (Bruker, Germany) were used to observe mineral concentrates. The research was done with the acceleration voltage of $25 \mathrm{kV}$, in a high vacuum. An electron microprobe CAMECA SX-100 (Cameca, Cedex, France), equipped with wave-length dispersive spectrometers (WDS), with the acceleration voltage of $15 \mathrm{kV}$ and current beam of $20 \mathrm{nA}$, was used for point analysis of minerals' chemical composition. Samples for SEM-BSE (Scanning Electron Microscopy-Backscattered Electron Mode) and EPMA (Electron probe microanalysis) analyses were coated with carbon. The EPMA analysis was performed on crystals TAP (thallium acid phthalate), LIF (lithium fluoride), PET (pentaerythritol) and LPET (large pentaerythritol). Reference material, detection limits and standard deviation are presented in Table 1. The research took place in the laboratories of the Department of Geology at the University of Warsaw.

Table 1. Conditions of EPMA (Electron probe microanalysis ) analyses.

\begin{tabular}{cccccc}
\hline Element & Standard & Analytical Line & Crystal & Detection Limit in wt\% & $\boldsymbol{\sigma}(\mathbf{w t} \%)$ \\
\hline $\mathbf{S i}$ & Diopside & $\mathrm{K} \alpha$ & $\mathrm{TAP}$ & 0.03 & $0.00-0.02$ \\
$\mathbf{T i}$ & Rutile & $\mathrm{K} \alpha$ & $\mathrm{LPET}$ & $0.02-0.03$ & 0.02 \\
$\mathbf{A l}$ & Orthoclase & $\mathrm{K} \alpha$ & $\mathrm{TAP}$ & 0.03 & $0.12-0.30$ \\
$\mathbf{F e}$ & $\mathrm{Fe}_{2} \mathrm{O}_{3}$ & $\mathrm{~K} \alpha$ & $\mathrm{LIF}$ & $0.10-0.12$ & $0.40-0.48$ \\
$\mathbf{V}$ & $\mathrm{V}_{2} \mathrm{O}_{5}$ & $\mathrm{~K} \alpha$ & $\mathrm{LIF}$ & $0.07-0.11$ & $0.07-0.08$ \\
$\mathbf{C r}$ & $\mathrm{Cr}_{2} \mathrm{O}_{3}$ & $\mathrm{~K} \alpha$ & $\mathrm{PET}$ & $0.06-0.08$ & $0.34-0.51$ \\
$\mathbf{M g}$ & Diopside & $\mathrm{K} \alpha$ & $\mathrm{TAP}$ & $0.02-0.03$ & $0.16-0.22$ \\
$\mathbf{C a}$ & Diopside & $\mathrm{K} \alpha$ & $\mathrm{PET}$ & $0.03-0.06$ & $0.00-0.03$ \\
$\mathbf{N a}$ & Albite & $\mathrm{K} \alpha$ & TAP & $0.07-0.10$ & $0.00-0.09$ \\
$\mathbf{K}$ & Orthoclase & $\mathrm{K} \alpha$ & PET & $0.02-0.05$ & $0.00-0.04$ \\
$\mathbf{M n}$ & Rhodonite & $\mathrm{K} \alpha$ & LIF & $0.11-0.12$ & $0.10-0.11$ \\
$\mathbf{C o}$ & CoO & $\mathrm{K} \alpha$ & LIF & $0.13-0.14$ & $0.12-0.13$ \\
$\mathbf{C u}$ & Cuprite & $\mathrm{K} \alpha$ & LIF & $0.15-0.22$ & $0.16-0.19$ \\
$\mathbf{Z n}$ & Sphalerite & $\mathrm{K} \alpha$ & LIF & $0.20-0.23$ & $0.18-0.19$ \\
$\mathbf{N i}$ & NiO & $\mathrm{K} \alpha$ & LIF & $0.11-0.14$ & $0.00-0.12$ \\
\hline
\end{tabular}

The chemical analysis of bulk samples was performed in a certified laboratory Bureau Veritas (Canada). Two analytical programs were used-LF202, where samples were melted with $\mathrm{Na}_{2} \mathrm{~B}_{4} \mathrm{O}_{7} /$ $\mathrm{Li}_{2} \mathrm{~B}_{4} \mathrm{O}$ and then dissolved with a mixture of aggressive acids and FA330 program for noble metals analysis with Fire Assay methodology (www.acmelab.com). The samples were tested using ICP-MS (Inductively coupled plasma mass spectrometry) and ICP-ES (Inductively Coupled Plasma Emission Spectroscopy) analyzers.

\section{The Geological Structure of the Area}

The geological structure (Figure 1) of New Guinea Island is a result of many complex geodynamic processes: Indo-Australian Plate and Pacific Plate collision [38-40], accretion of island arcs [31] subduction and exhumation of fragments of lithosphere plates [41-43], reversing of subduction polarization [44] and ophiolites obduction [45].

The geological structure of the Waropen Regency and Cendrawasih Bay is still poorly known (Figures 1 and 2). Contemporary geotectonic interpretation of the region indicates that this area is placed in the eastern part of the Melanesian collision arc [46] that was formed above the subduction 
zone $[42,47]$. Structurally, the Waropen region (Waropen Basin) and the adjacent Cendrawasih Bay are parts of the large North Basin (Irian). The history of the basement of the terranes still remains unsolved. Analysis of gravimetric anomalies indicates that the basement of sedimentary basin is represented by ocean crust rocks (ophiolite, diorite intrusions) and is covered with a sediment sequence about $6.5 \mathrm{~km}$ thick (Figure 4) [33-35]. The oldest analyzed sediments in this sedimentary basin are Paleocene and early-Oligocene rocks from the Awuewa formation. This sequence is discordantly covered by Miocene-Pliocene limestone of the Darante formation [34]. The consequence of the change in the depositional conditions was the development sedimentation of the Makatas formation. The rapid basin subsidence led to a discordant sedimentation, which occurred in almost the whole North Basin. The sediments of the Mamberamo formation are a result of erosion. It is composed of Pliocene-Pleistocene regressive-transgressive sediments that are regionally differentiated (four parts of the Mamberamo formation). The uppermost series of cluster sediments are Neogene deposits assotiated with a system of inflow cones and deltas [34]. Dickinson [48] suggests that the formation of the North Basin and the deposition of cluster deposits is a result of rapid subsidence of the structure (fore-arc basin) due to compression forces caused by the collision of the Indo-Australian and Pacific plates.

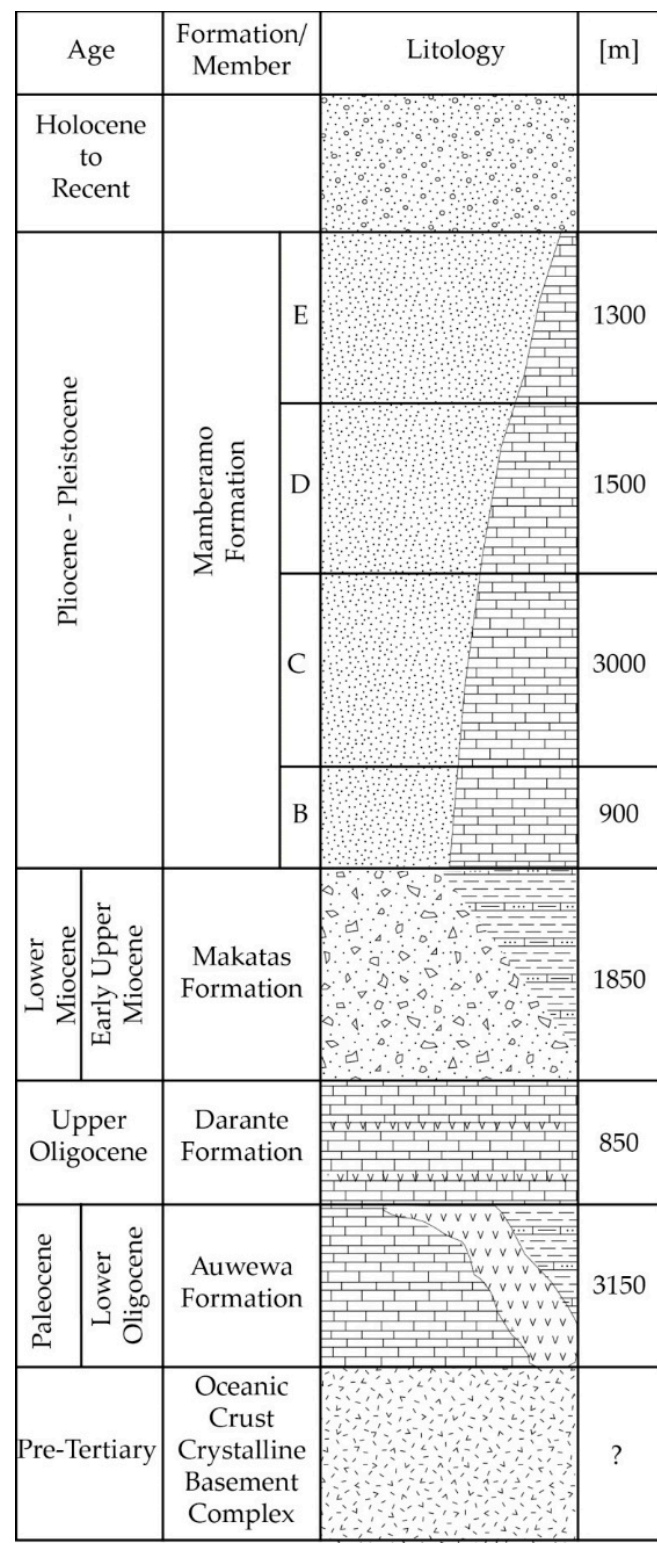

\section{Legend}

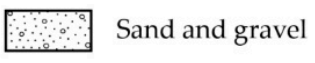

Clastic sediments

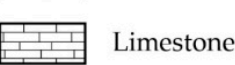

Conglomerate, wacks

Mudstone, schist

Limestone with local
mixed volcanic

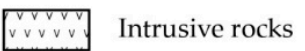

Ophiolite, basalt volcanics

Figure 4. Geological profile of Waropen Basin's sediments [33-35]. 


\section{Results of Analyses}

\subsection{Mineralogy and Geochemistry of the Beach Sediments}

The macroscopic observations of samples allowed evaluating and classifying the deposit by the color, grain size and the organic components' occurrence. The sieve analysis (Table 2) has shown that the beach sediments are sands (10 samples) and muddy sand (sample NMI 0115), grey and blackish-grey in color and moderately to well sorted. Skewness for the analyzed samples indicates a negative skew distribution and leptokurtic measure of flatness. Sand from sample NMI 0119 is the only poorly graded one. The heavy minerals concentrate samples have not been included in the sieve analysis. In the beach sediments, biogenic fragments of marine organisms were found (from $2.5 \mathrm{wt} \%$ to $23 \mathrm{wt} \%$ ) - mainly shells, corals, foraminifera and snails. All samples have a polymineral composition. On the Southern Region a beach sediment (Figure 3A,B) contain quartz, potassium feldspar, plagioclase, pyroxenes, amphiboles, epidotes, garnets, andalusite and illite, muscovite, biotite, serpentine, lyssarite (serpentine subgroup), zircon, monazite, xenotime, chromium spinel, magnetite, rutile and ilmenite. In the samples from the Northern Region are dominate mainly (Figure 3C,D): diopside, hornblende, riebeckite, magnetite, chromian spinel, olivine, serpentine subgroup, albite, sanidine, biotite and quartz. Samples from alluvial sediments of the Botawa River have a similar mineral composition. Based on microscopic analyses, two generations of grains with different levels of roundness have been separated: 1) moderate and well-rounded, discoidal and spherical grains (Figure 3) poorly rounded (Figure 5A), automorphic and elongated grains (Figure 5A,B). The samples contain numerous fragments of metamorphic rocks: chlorite, quartz and mica schists. The content of heavy minerals in the samples $(0.05-0.063 \mathrm{~mm}$ fraction size) varies from $2.4 \mathrm{wt} \%$ to $29 \mathrm{wt} \%$. The content of heavy minerals in $0.25 \mathrm{~mm}$ fraction in Northern Region is $34 \mathrm{wt} \%$ on average, while in the Southern Region equals $14 \mathrm{wt} \%$.

Table 2. Analysis of grain size and degree of sorting of beach sediment samples (in \%). Statistic parameters ( $\phi$ method [37]).

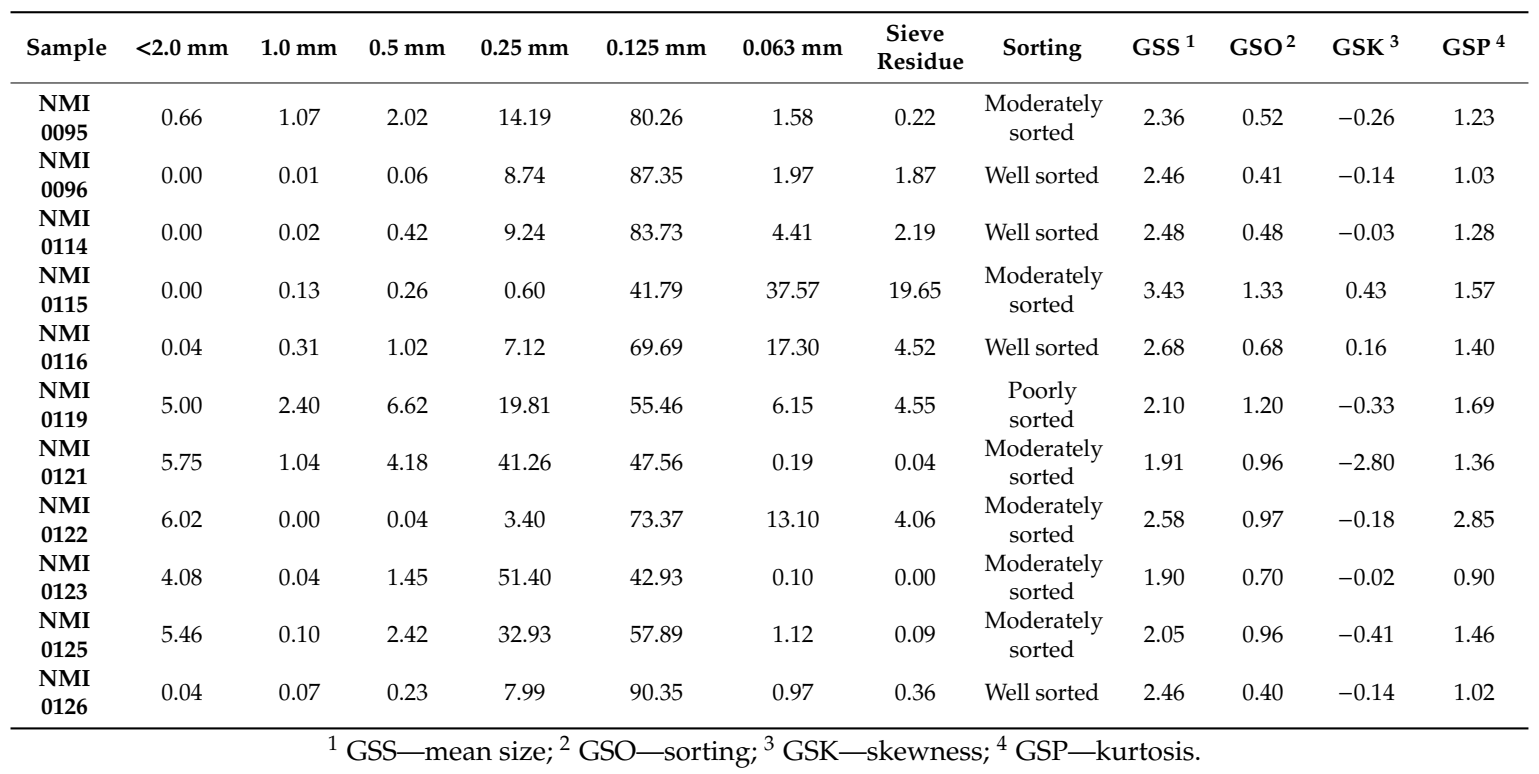

The chemical composition of beach sediments (Table 3) is relatively homogenous. The contents of $\mathrm{Cr}_{2} \mathrm{O}_{3}$ are high, ranging from $0.01 \mathrm{wt} \%$ to $1.17 \mathrm{wt} \%$. In the samples from the Northern Region, only higher contents of $\mathrm{CaO}$, from $2.74 \mathrm{wt} \%$ to $8.63 \mathrm{wt} \%$ are noted, while in the Southern Region, it is at the level of $1.29 \mathrm{wt} \%$ to $5.42 \mathrm{wt} \%$. The loss on ignition (LOI) in the samples is very high, measuring from $6.5 \%$ to $12.8 \%$. The highest concentration of trace elements in the samples is found for Ni, from 292 to 556 ppm, as well as for V 72-254 ppm. Lower contents were found for Zn 73-181 ppm, Co 24.1-55 ppm, $\mathrm{Pb}$ 5.7-21 ppm and Cu 5-12.6 ppm. The Fire Assay Analysis (FA330-Fire-Assay Analysis) results 
show very low contents of noble metals in the beach sediments. Usually, they are below the detection limit of the method used (ICP-MS). The maximum contents for Pt are up to $6 \mathrm{ppb}, \mathrm{Pd}$ up to $2 \mathrm{ppb}$ and Au up to $4 \mathrm{ppb}$. The higher concentration of Ag up to $6.1 \mathrm{ppm}$ is noted in the samples.
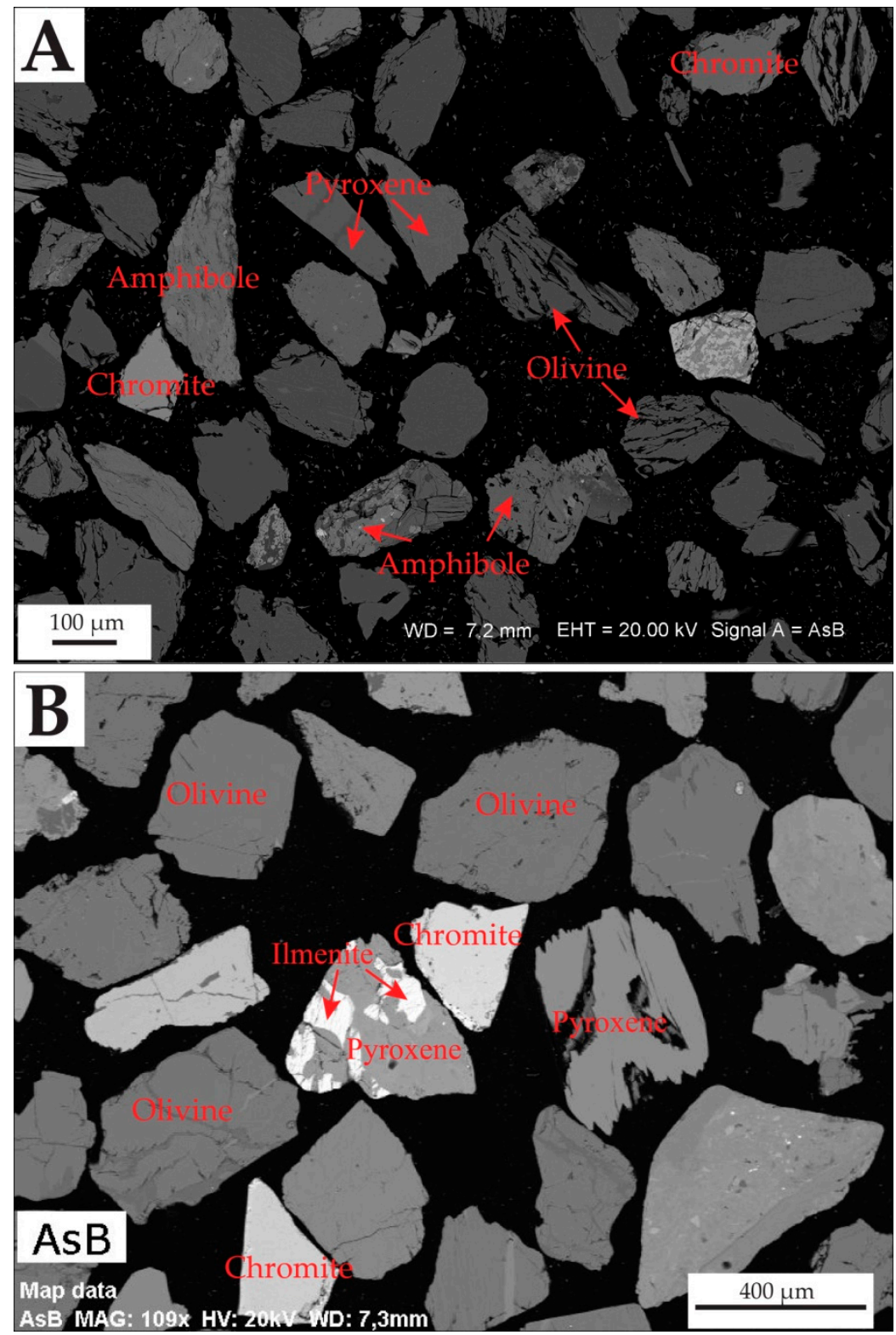

Figure 5. SEM-BSE (Scanning Electron Microscopy-Backscattered Electron Mode) images of two heavy mineral concentrates from beach sediment samples. (A) poorly sorted sediments, (B) well sorted sediments.

\subsection{Mineralogy and Geochemistry of Alluvial Sediments from Botawa River}

Mineral analysis of the heavy minerals concentrate from the Botawa River sediments shows the linear growth of metals contents in terrigenous sediments, from the coast to the mountain source area (Table 4). There are abnormally high contents of $\mathrm{Cr}_{2} \mathrm{O}_{3}$ up to $24.83 \%$ (Figure 6), $\mathrm{TiO}_{2}$ up to 3.93\%, $\mathrm{Ni}$ up to $492.6 \mathrm{ppm}$ and V up to $2026 \mathrm{ppm}$. Heavy minerals concentrate is composed of chromite, magnetite, ilmenite, pyroxenes, amphiboles, olivine and secondly quartz, plagioclases and chlorite. In sample WAR 31, a very high content of noble metals is revealed: Au up to 3.05 ppm, Pd up to 11 ppb and Pt up to $8 \mathrm{ppb}$. 
Table 3. Chemical composition of beach sediment samples.

\begin{tabular}{|c|c|c|c|c|c|c|c|c|c|c|c|}
\hline \multirow{2}{*}{ Sample } & \multicolumn{5}{|c|}{ Southern Region } & \multicolumn{6}{|c|}{ Northern Region } \\
\hline & $\begin{array}{l}\text { NMI } \\
0095\end{array}$ & $\begin{array}{l}\text { NMI } \\
0096\end{array}$ & $\begin{array}{l}\text { NMI } \\
0115\end{array}$ & $\begin{array}{l}\text { NMI } \\
0116\end{array}$ & $\begin{array}{l}\text { NMI } \\
0126\end{array}$ & $\begin{array}{l}\text { NMI } \\
0114\end{array}$ & $\begin{array}{l}\text { NMI } \\
0119\end{array}$ & $\begin{array}{l}\text { NMI } \\
0121\end{array}$ & $\begin{array}{l}\text { NMI } \\
0122\end{array}$ & $\begin{array}{l}\text { NMI } \\
0123\end{array}$ & $\begin{array}{l}\text { NMI } \\
0125\end{array}$ \\
\hline \multicolumn{12}{|c|}{ Oxides in wt $\%$} \\
\hline $\mathrm{SiO}_{2}$ & 58.83 & 61.22 & 62.94 & 59.99 & 62.17 & 57.78 & 52.37 & 53.77 & - & - & - \\
\hline $\mathrm{Al}_{2} \mathrm{O}_{3}$ & 10.49 & 8.84 & 11.34 & 10.62 & 7.99 & 12.00 & 9.51 & 8.31 & - & - & - \\
\hline $\mathrm{Fe}_{2} \mathrm{O}_{3}$ & 8.37 & 6.60 & 6.53 & 6.67 & 4.52 & 6.73 & 5.63 & 7.89 & - & - & - \\
\hline $\mathrm{MgO}$ & 8.99 & 10.61 & 6.28 & 8.04 & 8.10 & 7.30 & 7.12 & 13.32 & - & - & - \\
\hline $\mathrm{CaO}$ & 1.29 & 1.85 & 1.69 & 2.44 & 5.42 & 2.74 & 8.63 & 6.29 & - & - & - \\
\hline $\mathrm{Na}_{2} \mathrm{O}$ & 1.77 & 1.55 & 1.73 & 1.72 & 1.57 & 1.90 & 1.67 & 1.17 & - & - & - \\
\hline $\mathrm{K}_{2} \mathrm{O}$ & 1.03 & 1.06 & 1.34 & 1.26 & 0.90 & 1.46 & 1.15 & 0.67 & - & - & - \\
\hline $\mathrm{TiO}_{2}$ & 0.89 & 0.34 & 0.63 & 0.53 & 0.38 & 0.64 & 0.49 & 0.74 & - & - & - \\
\hline $\mathrm{P}_{2} \mathrm{O}_{5}$ & 0.21 & 0.14 & 0.14 & 0.14 & 0.12 & 0.15 & 0.11 & 0.11 & - & - & - \\
\hline $\mathrm{MnO}$ & 0.11 & 0.06 & 0.09 & 0.09 & 0.06 & 0.08 & 0.08 & 0.15 & - & - & - \\
\hline $\mathrm{Cr}_{2} \mathrm{O}_{3}$ & 1.17 & 0.93 & 0.16 & 0.14 & 0.14 & 0.01 & 0.11 & 0.66 & - & - & - \\
\hline LOI & 6.50 & 7.90 & 6.90 & 8.10 & 8.30 & 8.80 & 12.80 & 6.50 & - & - & - \\
\hline Total & 99.78 & 99.81 & 99.86 & 99.83 & 99.83 & 99.85 & 99.84 & 99.70 & - & - & - \\
\hline \multicolumn{12}{|c|}{ Element in ppm } \\
\hline $\mathrm{Ni}$ & 437 & 507 & 292 & 379 & 320 & 344 & 315 & 556 & 334 & 350 & 446 \\
\hline Co & 29.4 & 45.9 & 32.1 & 30.7 & 24.1 & 28.7 & 25.6 & 42 & 28 & 26 & 55 \\
\hline Mo & 0.3 & 0.2 & 0.2 & 0.3 & 0.2 & 0.3 & 0.4 & 2.7 & 2.6 & 2.5 & 1.6 \\
\hline $\mathrm{Pb}$ & 21 & 17.3 & 18.2 & 17.5 & 8.7 & 17.3 & 14.1 & 5.7 & 15.6 & 8.5 & 11.7 \\
\hline $\mathrm{Cu}$ & 7.2 & 10.2 & 9.9 & 10.1 & 5.0 & 12.2 & 9.4 & 7.1 & 7.4 & 8.8 & 12.6 \\
\hline Zn & 76 & 178 & 82 & 95 & 88 & 99 & 79 & 106 & 82 & 73 & 181 \\
\hline Sn & 1.2 & 2.5 & 1.8 & 1.6 & 1.4 & 1.9 & 1.4 & 1.4 & 1.8 & 1.9 & 1.6 \\
\hline $\mathbf{V}$ & 72 & 206 & 92 & 104 & 84 & 110 & 91 & 150 & 98 & 98 & 254 \\
\hline $\mathbf{W}$ & 0.4 & 0.6 & 0.5 & 0.7 & 0.4 & 0.7 & 0.6 & $<0.5$ & $<0.5$ & $<0.5$ & $<0.5$ \\
\hline Ag & 0.1 & 2.9 & 2.3 & 2.4 & 6.1 & 2.0 & 2.8 & $<0.5$ & $<0.5$ & $<0.5$ & $<0.5$ \\
\hline \multicolumn{12}{|c|}{ Element in ppb } \\
\hline $\mathrm{Au}$ & 3 & 4 & 3 & 4 & 4 & 3 & 3 & 3 & 4 & 3 & 3 \\
\hline $\mathbf{P t}$ & $<3$ & $<3$ & $<3$ & $<3$ & 6 & $<3$ & $<3$ & $<3$ & $<3$ & $<3$ & $<3$ \\
\hline Pd & $<2$ & 2 & $<3$ & $<2$ & $<2$ & $<2$ & $<2$ & $<2$ & $<2$ & $<2$ & $<2$ \\
\hline
\end{tabular}

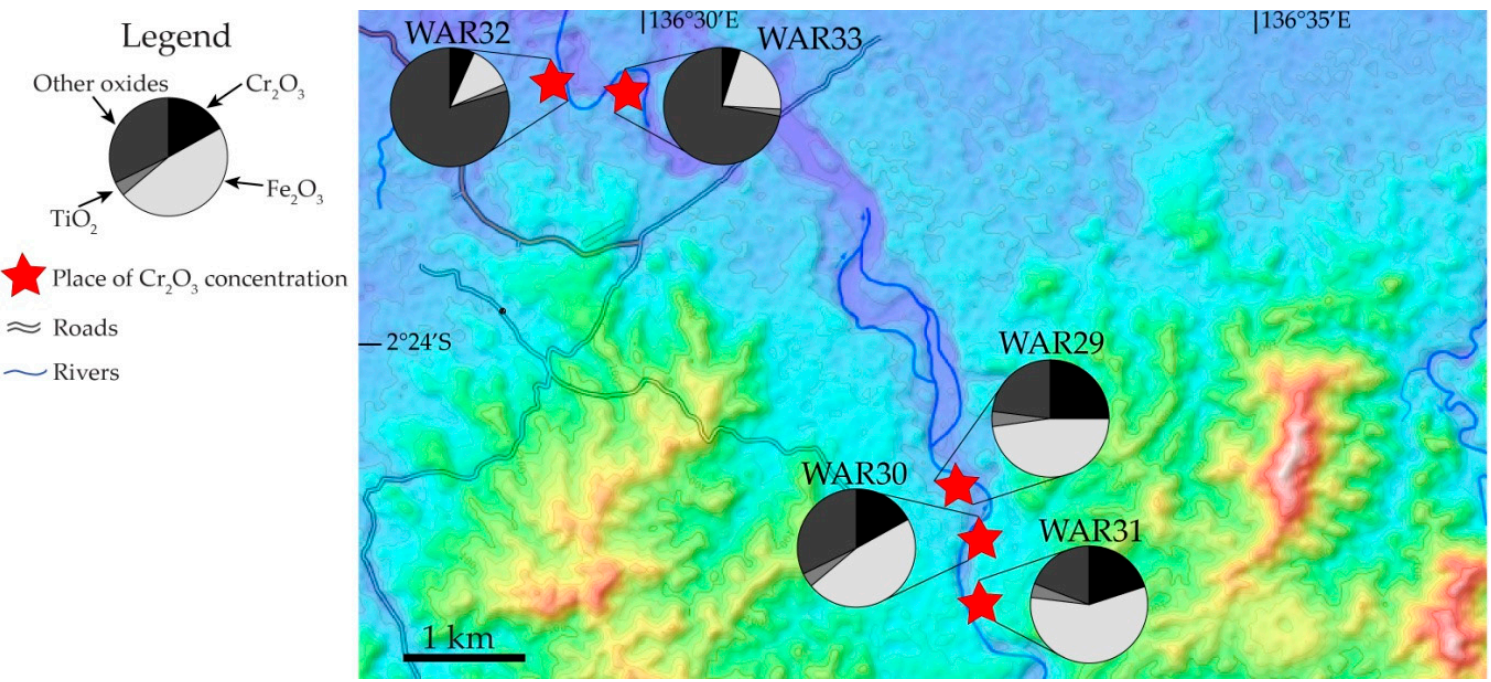

Figure 6. The concentration of oxides $\mathrm{Cr}_{2} \mathrm{O}_{3}, \mathrm{TiO}_{2}$ and $\mathrm{FeO}_{2}$ in samples. Sample location in the upper Botawa River is shown in Figure 2. 
Table 4. Chemical composition of heavy minerals concentrate from the Botawa River. ICP-MS (Inductively coupled plasma mass spectrometry) analysis.

\begin{tabular}{cccccc}
\hline Sample & WAR 32 & WAR 33 & WAR 29 & WAR 30 & WAR 31 \\
\hline \multicolumn{5}{c}{ Oxide in wt $\%$. } \\
\hline $\mathbf{C r}_{\mathbf{2}} \mathbf{O}_{\mathbf{3}}$ & 6.69 & 4.76 & 24.83 & 17.19 & 20.25 \\
$\mathbf{F e}_{\mathbf{2}} \mathbf{O}_{\mathbf{3}}$ & 11.88 & 21.42 & 48.01 & 47.18 & 56.83 \\
$\mathbf{T i O}_{\mathbf{2}}$ & 1.68 & 1.68 & 3.67 & 3.66 & 3.93 \\
\hline \multicolumn{7}{c}{ Element in ppm } \\
\hline $\mathbf{M o}$ & 5.9 & 3.2 & 5.0 & 4.1 & 2.7 \\
$\mathbf{C u}$ & 12.0 & 21.1 & 37.3 & 38.3 & 36.9 \\
$\mathbf{P b}$ & 14.0 & 6.1 & 15.3 & 5.8 & 62.4 \\
$\mathbf{Z n}$ & 409 & 431 & 1247 & 1004 & 1045 \\
$\mathbf{N i}$ & 237.7 & 329.1 & 492.6 & 461.5 & 411.7 \\
$\mathbf{C o}$ & 95 & 98 & 321 & 252 & 265 \\
$\mathbf{S n}$ & 1.3 & 1.9 & 1.6 & 1.5 & 2.2 \\
$\mathbf{V}$ & 404 & 681 & 1786 & 1710 & 2026 \\
$\mathbf{W}$ & $<0.5$ & $<0.5$ & $<0.5$ & $<0.5$ & $<0.5$ \\
$\mathbf{A g}$ & $<0.5$ & $<0.5$ & $<0.5$ & 4.7 & $<0.5$ \\
$\mathbf{A u}$ & - & - & - & - & 3.05 \\
$\mathbf{P t} \mathbf{( p p b )}$ & - & - & - & - & 8 \\
$\mathbf{P d}$ & - & - & - & - & 11 \\
\hline
\end{tabular}

\subsection{Indicator Minerals}

\subsubsection{Chromian Spinel}

Chromian spinels occur in all grain fractions of the beach sediments. The dominant grain class is the 0.5-0.063 $\mathrm{mm}$ fraction. The minerals rarely form isometric crystals. Normally, they are allotriomorphic. Chromian spinels occur in paragenesis with magnetite and serpentinized olivine. Some of the grains were transformed during the post-magmatic processes in low-grade metamorphic processes related to serpentinization (Figure 7A). The micro-area analysis (EPMA) of minerals shows significant differences in the chemical composition (Table 5). Chromian spinels form a continuous spinel-magnesiochromitechromite series (Figure 8). Magnesiochromite is the dominant group and the differentiation of $\mathrm{Cr}$, $\mathrm{Al}$ and Fe contents is visible in it. Two groups of chromian spinels have been separated based on changes in the $\mathrm{Cr} \#$ index $\mathrm{Cr} \#=100 \times \mathrm{Cr} /(\mathrm{Cr}+\mathrm{Al})$ [49]: high-Al spinel (Cr\# 20-60) and high-Cr spinel $(\mathrm{Cr} \#=60-80)$.
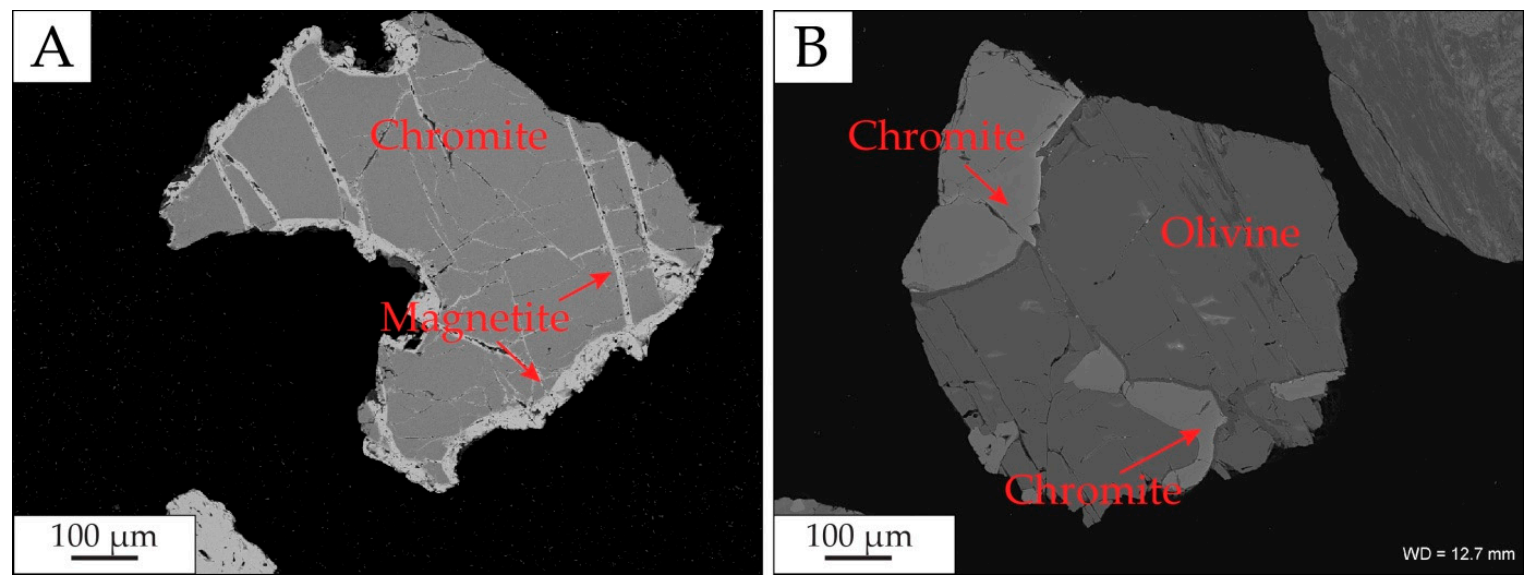

Figure 7. Chromite paragenesis with magnetite. SEM-BSE (Scanning Electron Microscopy-Backscattered Electron Mode) image. (A) single grain with magnetite, (B) olivine grain with chromite. 


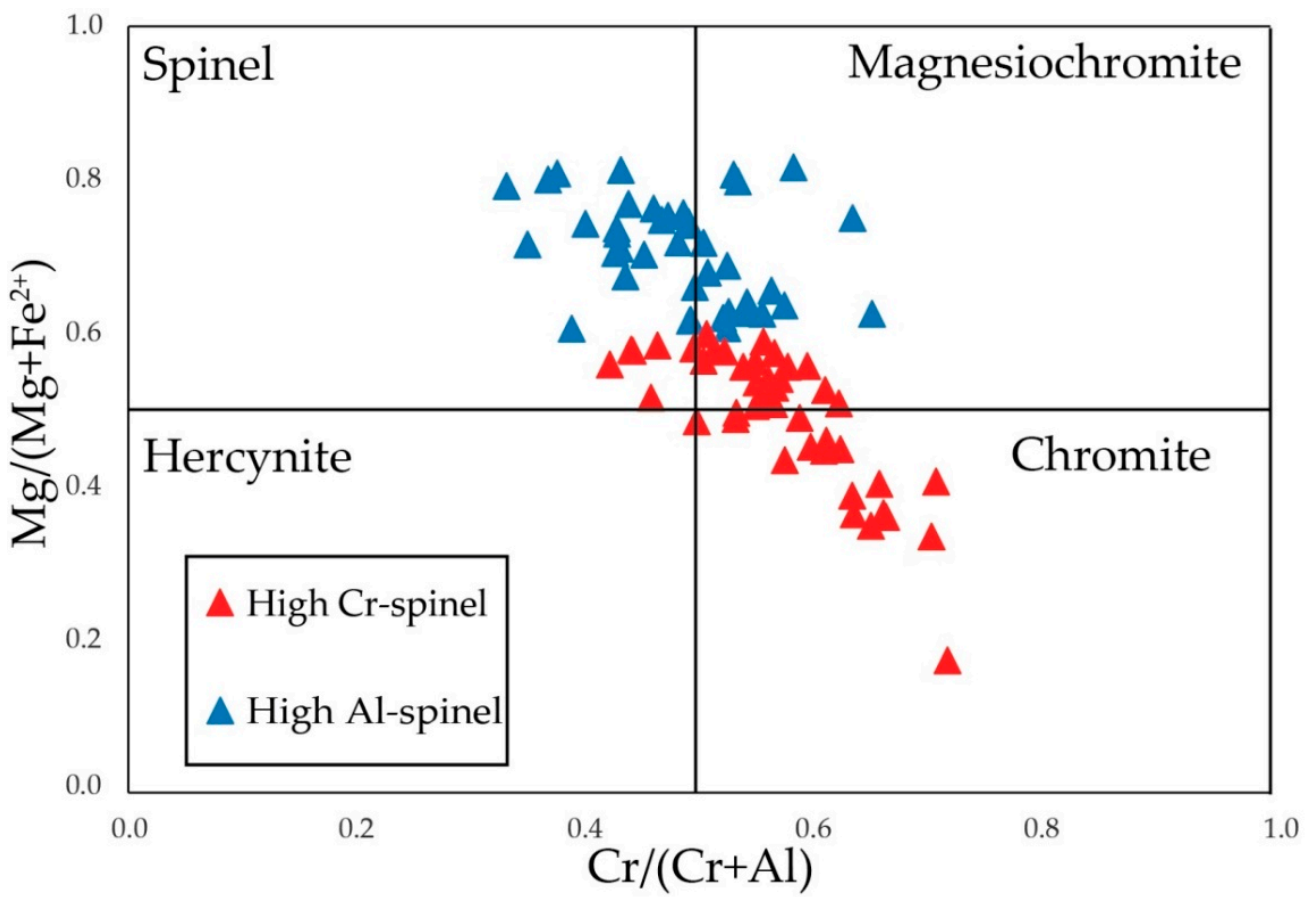

Figure 8. Classification of the composition of chromian spinels from the Waropen Regency (after [50]).

The $\mathrm{Cr}_{2} \mathrm{O}_{3}$ content in the high-Cr spinels varies from $46.03 \mathrm{wt} \%$ to $61.79 \mathrm{wt} \%$, with high $\mathrm{Cr} \# 61-82$, contrary to high-Al spinels where $\mathrm{Cr}_{2} \mathrm{O}_{3}$ is from $29.34 \mathrm{wt} \%$ to $46.69 \mathrm{wt} \%$ and $\mathrm{Cr} \# 33-59$. All spinel grains contain a low amount of $\mathrm{TiO}_{2}<0.6 \mathrm{wt} \%$. High-Al chromian spinels show a wide range of chemical composition- $\mathrm{MgO}\left(8.75 \mathrm{wt}^{\mathrm{T}} \%-17.46 \mathrm{wt} \%\right), \mathrm{FeO}(13.31 \mathrm{wt} \%-29.54 \mathrm{wt} \%), \mathrm{MnO}(0.11 \mathrm{wt} \%-0.42 \mathrm{wt} \%)$. Almost similar contents are present in the high-Cr spinels: $\mathrm{MgO}(6.37 \mathrm{wt} \%-14.10 \mathrm{wt} \%), \mathrm{FeO}(13.70 \mathrm{wt} \%-27.69 \mathrm{wt} \%)$, $\mathrm{MnO}(0.19 \mathrm{wt} \%-0.47 \mathrm{wt} \%)$. In Cr- and Al-spinels, there is enrichment in transition metals: $\mathrm{Co}, \mathrm{Ni}, \mathrm{Zn}$ and $\mathrm{Cu}$ (Table 5). Microscopic observations (SEM-BSE) have revealed the presence of olivine and chromite (Figure 7B) intergrowths but it has not revealed the alloys of noble metals.

\subsubsection{Olivine}

The microscopic observations of beach sediments have allowed separating two groups of olivine: 1) yellow olivine grains, under the process or already after the process of serpentinization; and 2) green olivine grains without any visible changes. Those minerals dominate in the $0.5-0.063 \mathrm{~mm}$ grain class. The fraction $>0.5 \mathrm{~mm}$ contains no olivine. The olivine serpentinization was accompanied by the development of a crack system, transformation of grains and a growth of new phases-magnetite. The micro-area analysis (Table 6) indicates that olivine is represented by magnesium varieties-forsterite (Fo 89.46-91.92). The magnesium index of olivine $\mathrm{Mg} \#=\mathrm{Mg} /(\mathrm{Mg}+\mathrm{Fe})$ is $90-92$. The characteristic feature of the grains is their stable concentration of $\mathrm{SiO}_{2}(40.59 \mathrm{wt} \%-41.41 \mathrm{wt} \%)$ and $\mathrm{MgO}(48.54 \mathrm{wt} \%-$ $50.37 \mathrm{wt} \%$ ). The $\mathrm{NiO}$ content varies from $0.29 \mathrm{wt} \%$ to $0.45 \mathrm{wt} \%$, which is typical for olivine originating from the Earth's mantle. The contents of $\mathrm{TiO}_{2}, \mathrm{Cr}_{2} \mathrm{O}_{3}, \mathrm{Al}_{2} \mathrm{O}_{3}$ are below detection limits. 
Table 5. Chemical composition of chromian spinel in $\mathrm{w} t \%$. EPMA (Electron probe microanalysis) analysis.

\begin{tabular}{|c|c|c|c|c|c|c|c|c|c|c|c|c|c|c|c|c|}
\hline \multirow{2}{*}{ Sample Oxide in $w t \%$} & \multicolumn{8}{|c|}{ High-Cr Spinels } & \multicolumn{8}{|c|}{ High-Al Spinels } \\
\hline & $1 / 1$ & 4/1. & 98/1. & $125 / 12$ & $125 / 27$ & $125 / 28$ & $125 / 39$ & $125 / 47$ & 6/1. & 7/1. & 21/1. & $25 / .1$ & $42 / 1$ & $44 / 1$. & $45 / 1$. & 46/1. \\
\hline $\mathrm{SiO}_{2}$ & 0.00 & 0.00 & 0.01 & 0.06 & 0.04 & 0.00 & 0.00 & 0.04 & 0.00 & 0.00 & 0.04 & 0.02 & 0.02 & 0.03 & 0.01 & 0.05 \\
\hline $\mathrm{TiO}_{2}$ & 0.15 & 0.19 & 0.12 & 0.12 & 0.21 & 0.09 & 0.12 & 0.09 & 0.11 & 0.12 & 0.15 & 0.07 & 0.44 & 0.07 & 0.19 & 0.08 \\
\hline $\mathrm{Al}_{2} \mathrm{O}_{3}$ & 17.32 & 13.87 & 12.20 & 9.73 & 9.31 & 13.83 & 12.87 & 16.81 & 26.11 & 20.75 & 21.86 & 23.30 & 20.91 & 33.45 & 26.27 & 28.46 \\
\hline $\mathrm{Fe}_{2} \mathrm{O}_{3}$ & 2.64 & 3.22 & 3.53 & 1.86 & 2.53 & 7.67 & 2.14 & 0.56 & 3.72 & 1.46 & 3.35 & 1.67 & 4.12 & 2.71 & 1.18 & 1.15 \\
\hline $\mathrm{V}_{2} \mathrm{O}_{3}$ & 0.18 & 0.31 & 0.22 & 0.11 & 0.21 & 0.20 & 0.26 & 0.32 & 0.26 & 0.30 & 0.31 & 0.24 & 0.26 & 0.12 & 0.18 & 0.20 \\
\hline $\mathrm{Cr}_{2} \mathrm{O}_{3}$ & 50.01 & 51.94 & 55.36 & 60.33 & 58.30 & 49.00 & 55.70 & 52.80 & 39.96 & 47.10 & 44.31 & 44.70 & 43.62 & 33.64 & 43.31 & 40.57 \\
\hline $\mathrm{FeO}$ & 18.41 & 22.78 & 18.97 & 16.99 & 21.77 & 20.29 & 18.28 & 18.29 & 16.88 & 18.44 & 18.38 & 17.18 & 20.17 & 13.82 & 15.36 & 16.19 \\
\hline $\mathrm{MgO}$ & 10.17 & 6.87 & 9.54 & 10.75 & 7.35 & 8.45 & 9.88 & 10.57 & 12.33 & 10.55 & 10.85 & 11.74 & 9.77 & 14.88 & 13.50 & 12.95 \\
\hline $\mathrm{CaO}$ & 0.01 & 0.04 & 0.01 & 0.00 & 0.02 & 0.01 & 0.01 & 0.00 & 0.01 & 0.01 & 0.02 & 0.00 & 0.00 & 0.02 & 0.04 & 0.05 \\
\hline $\mathrm{MnO}$ & 0.31 & 0.45 & 0.31 & 0.23 & 0.34 & 0.33 & 0.20 & 0.28 & 0.23 & 0.28 & 0.23 & 0.25 & 0.30 & 0.22 & 0.20 & 0.19 \\
\hline $\mathrm{CoO}$ & 0.00 & 0.00 & 0.13 & 0.11 & 0.08 & 0.05 & 0.00 & 0.00 & 0.00 & 0.00 & 0.04 & 0.07 & 0.05 & 0.06 & 0.02 & 0.20 \\
\hline $\mathrm{CuO}$ & 0.00 & 0.00 & 0.08 & 0.00 & 0.00 & 0.00 & 0.00 & 0.00 & 0.00 & 0.00 & 0.10 & 0.02 & 0.00 & 0.14 & 0.00 & 0.15 \\
\hline $\mathrm{ZnO}$ & 0.41 & 0.34 & 0.15 & 0.09 & 0.22 & 0.37 & 0.00 & 0.01 & 0.15 & 0.17 & 0.14 & 0.13 & 0.23 & 0.11 & 0.19 & 0.19 \\
\hline $\mathrm{NiO}$ & 0.03 & 0.00 & 0.01 & 0.06 & 0.09 & 0.13 & 0.03 & 0.09 & 0.12 & 0.06 & 0.10 & 0.10 & 0.04 & 0.17 & 0.10 & 0.15 \\
\hline Total & 99.67 & 100.08 & 100.64 & 100.43 & 100.49 & 100.50 & 99.60 & 99.84 & 99.87 & 99.27 & 99.90 & 99.47 & 99.91 & 99.42 & 100.55 & 100.59 \\
\hline \multicolumn{17}{|c|}{ apfu } \\
\hline $\mathrm{Si}$ & 0.00 & 0.00 & 0.00 & 0.01 & 0.01 & 0.00 & 0.00 & 0.01 & 0.00 & 0.00 & 0.01 & 4.35 & 0.00 & 0.01 & 0.00 & 0.01 \\
\hline $\mathrm{Ti}$ & 0.03 & 0.04 & 0.02 & 0.02 & 0.04 & 0.02 & 0.02 & 0.02 & 0.02 & 0.02 & 0.03 & 0.01 & 0.08 & 0.01 & 0.03 & 0.02 \\
\hline $\mathrm{Al}$ & 5.25 & 4.34 & 3.76 & 3.01 & 2.95 & 4.28 & 3.98 & 5.08 & 7.51 & 6.20 & 6.46 & 6.82 & 6.25 & 9.23 & 7.45 & 8.03 \\
\hline $\mathrm{Fe}^{3+}$ & 0.51 & 0.64 & 0.70 & 0.37 & 0.51 & 1.52 & 0.42 & 0.11 & 0.68 & 0.28 & 0.63 & 0.31 & 0.79 & 0.48 & 0.21 & 0.21 \\
\hline $\mathrm{V}$ & 0.04 & 0.07 & 0.05 & 0.02 & 0.05 & 0.04 & 0.06 & 0.07 & 0.05 & 0.06 & 0.06 & 0.05 & 0.05 & 0.02 & 0.04 & 0.04 \\
\hline $\mathrm{Cr}$ & 10.16 & 10.91 & 11.45 & 12.52 & 12.41 & 10.17 & 11.55 & 10.70 & 7.71 & 9.44 & 8.78 & 8.78 & 8.74 & 6.23 & 8.24 & 7.67 \\
\hline $\mathrm{Fe}^{2+}$ & 3.96 & 5.06 & 4.15 & 3.73 & 4.90 & 4.46 & 4.01 & 3.92 & 3.45 & 3.91 & 3.85 & 3.57 & 4.28 & 2.71 & 3.09 & 3.24 \\
\hline $\mathrm{Mg}$ & 3.90 & 2.72 & 3.72 & 4.21 & 2.95 & 3.31 & 3.86 & 4.04 & 4.49 & 3.99 & 4.05 & 4.35 & 3.69 & 5.20 & 4.84 & 4.62 \\
\hline $\mathrm{Ca}$ & 0.00 & 0.01 & 0.00 & 0.00 & 0.00 & 0.00 & 0.00 & 0.00 & 0.00 & 0.00 & 0.00 & 0.00 & 0.00 & 0.00 & 0.01 & 0.01 \\
\hline Mn & 0.07 & 0.10 & 0.07 & 0.05 & 0.08 & 0.07 & 0.05 & 0.06 & 0.05 & 0.06 & 0.05 & 0.05 & 0.07 & 0.04 & 0.04 & 0.04 \\
\hline Co & 0.00 & 0.00 & 0.03 & 0.02 & 0.02 & 0.01 & 0.00 & 0.00 & 0.00 & 0.00 & 0.01 & 0.01 & 0.01 & 0.01 & 0.00 & 0.04 \\
\hline $\mathrm{Cu}$ & 0.00 & 0.00 & 0.02 & 0.00 & 0.00 & 0.00 & 0.00 & 0.00 & 0.00 & 0.00 & 0.02 & 0.00 & 0.00 & 0.02 & 0.00 & 0.03 \\
\hline $\mathrm{Zn}$ & 0.08 & 0.07 & 0.03 & 0.02 & 0.04 & 0.07 & 0.00 & 0.00 & 0.03 & 0.03 & 0.03 & 0.02 & 0.04 & 0.02 & 0.03 & 0.03 \\
\hline $\mathrm{Ni}$ & 0.00 & 0.00 & 0.00 & 0.01 & 0.01 & 0.01 & 0.00 & 0.01 & 0.01 & 0.01 & 0.01 & 0.01 & 0.00 & 0.01 & 0.01 & 0.01 \\
\hline Cr\# & 0.66 & 0.72 & 0.75 & 0.81 & 0.81 & 0.70 & 0.74 & 0.68 & 0.51 & 0.60 & 0.58 & 0.56 & 0.58 & 0.40 & 0.53 & 0.49 \\
\hline Mg\# & 0.50 & 0.35 & 0.47 & 0.53 & 0.38 & 0.43 & 0.49 & 0.51 & 0.57 & 0.50 & 0.51 & 0.55 & 0.46 & 0.66 & 0.61 & 0.59 \\
\hline $\mathrm{Fe}^{3+\#}$ & 0.03 & 0.04 & 0.04 & 0.02 & 0.03 & 0.09 & 0.03 & 0.01 & 0.04 & 0.02 & 0.04 & 0.02 & 0.05 & 0.03 & 0.01 & 0.01 \\
\hline $\mathrm{Cr}$ & 63.83 & 68.63 & 71.98 & 78.76 & 78.17 & 63.71 & 72.42 & 67.35 & 48.49 & 59.31 & 55.33 & 55.17 & 55.42 & 39.08 & 51.81 & 48.24 \\
\hline Al & 32.95 & 27.32 & 23.64 & 18.93 & 18.61 & 26.80 & 24.94 & 31.96 & 47.22 & 38.94 & 40.68 & 42.88 & 39.59 & 57.92 & 46.85 & 50.46 \\
\hline $\mathrm{Fe}^{3+}$ & 3.22 & 4.05 & 4.37 & 2.31 & 3.23 & 9.49 & 2.65 & 0.68 & 4.29 & 1.75 & 3.98 & 1.96 & 4.98 & 2.99 & 1.35 & 1.30 \\
\hline $\mathrm{FeO} / \mathrm{MgO}$ & 1.81 & 3.32 & 1.99 & 1.58 & 2.96 & 2.40 & 1.85 & 1.73 & 1.37 & 1.75 & 1.69 & 1.46 & 2.06 & 0.93 & 1.14 & 1.25 \\
\hline $\mathrm{Cr} / \mathrm{Fe}$ & 4.55 & 3.83 & 4.72 & 6.11 & 4.59 & 3.40 & 5.21 & 5.31 & 3.73 & 4.51 & 3.92 & 4.53 & 3.45 & 3.91 & 4.99 & 4.50 \\
\hline \multicolumn{17}{|c|}{ Estimated composition for parental magmas } \\
\hline $\mathrm{Al}_{2} \mathrm{O}_{3}$ melt & 13.78 & 12.62 & 11.95 & 10.76 & 10.53 & 12.60 & 12.23 & 13.62 & 15.78 & 14.83 & 15.04 & 15.31 & 14.86 & 16.80 & 15.80 & 16.13 \\
\hline $\mathrm{TiO}_{2 \text { melt }}$ & 0.25 & 0.29 & 0.12 & 0.22 & 0.31 & 0.18 & 0.22 & 0.19 & 0.08 & 0.12 & 0.29 & - & 0.44 & - & 0.46 & - \\
\hline $\mathrm{FeO} / \mathrm{MgO}_{\text {melt }}$ & 0.46 & 0.99 & 0.44 & 0.18 & 0.79 & 0.63 & 0.40 & 0.42 & 0.32 & 0.49 & 0.47 & 0.36 & 0.65 & 0.06 & 0.15 & 0.28 \\
\hline
\end{tabular}


Table 6. Chemical composition of olivine in wt $\%$. EPMA analysis.

\begin{tabular}{|c|c|c|c|c|c|c|c|c|c|c|c|c|c|c|c|c|}
\hline & \multicolumn{16}{|c|}{ Sample } \\
\hline & $1 / 1$ & $13 / 1$ & $14 / 1$ & $18 / 1$ & 19/1. & $24 / 1$ & $57 / 1$. & $58 / 1$. & $59 / 1$. & $65 / 1$ & $66 / 1$ & $2 / 2$ & $4 / 2$ & $6 / 7$ & $8 / 9$. & $41 / 10$ \\
\hline & \multicolumn{16}{|c|}{ wt $\%$} \\
\hline $\mathrm{SiO}_{2}$ & 41.14 & 40.94 & 41.09 & 41.13 & 41.04 & 41.42 & 41.16 & 40.59 & 41.10 & 41.07 & 41.10 & 40.73 & 40.42 & 40.56 & 41.22 & 40.63 \\
\hline $\mathrm{TiO}_{2}$ & 0.01 & 0.02 & 0.02 & 0.02 & 0.03 & 0.00 & 0.01 & 0.02 & 0.02 & 0.00 & 0.00 & 0.00 & 0.00 & 0.00 & 0.00 & 0.00 \\
\hline $\mathrm{Al}_{2} \mathrm{O}_{3}$ & 0.00 & 0.00 & 0.00 & 0.00 & 0.01 & 0.00 & 0.00 & 0.00 & 0.00 & 0.00 & 0.00 & 0.00 & 0.00 & 0.00 & 0.00 & 0.00 \\
\hline $\mathrm{Cr}_{2} \mathrm{O}_{3}$ & 0.01 & 0.01 & 0.00 & 0.01 & 0.00 & 0.00 & 0.04 & 0.03 & 0.00 & 0.00 & 0.00 & 0.01 & 0.00 & 0.03 & 0.00 & 0.00 \\
\hline $\mathrm{FeO}$ & 10.11 & 10.04 & 9.83 & 9.96 & 8.98 & 7.76 & 9.31 & 9.91 & 8.82 & 9.89 & 9.23 & 9.56 & 10.21 & 9.74 & 9.87 & 10.34 \\
\hline $\mathrm{MnO}$ & 0.09 & 0.15 & 0.11 & 0.11 & 0.12 & 0.13 & 0.19 & 0.17 & 0.14 & 0.12 & 0.10 & 0.17 & 0.09 & 0.16 & 0.11 & 0.09 \\
\hline $\mathrm{MgO}$ & 48.84 & 48.54 & 48.67 & 48.76 & 49.89 & 50.37 & 49.65 & 48.70 & 49.80 & 49.22 & 49.32 & 48.98 & 49.26 & 48.54 & 48.89 & 48.56 \\
\hline $\mathrm{CaO}$ & 0.04 & 0.02 & 0.02 & 0.17 & 0.02 & 0.05 & 0.03 & 0.03 & 0.01 & 0.02 & 0.02 & 0.01 & 0.02 & 0.01 & 0.03 & 0.02 \\
\hline $\mathrm{NiO}$ & 0.39 & 0.29 & 0.41 & 0.32 & 0.45 & 0.41 & 0.32 & 0.42 & 0.45 & 0.37 & 0.33 & 0.21 & 0.18 & 0.12 & 0.20 & 0.30 \\
\hline \multirow[t]{2}{*}{ Total } & 100.62 & 99.99 & 100.15 & 100.50 & 100.54 & 100.13 & 100.70 & 99.87 & 100.35 & 100.68 & 100.10 & 99.67 & 100.18 & 99.18 & 100.31 & 99.94 \\
\hline & \multicolumn{16}{|c|}{ apfu } \\
\hline $\mathrm{Si}$ & 1.004 & 1.004 & 1.006 & 1.005 & 0.998 & 1.005 & 1.000 & 0.998 & 1.000 & 1.001 & 1.004 & 1.001 & 0.992 & 1.002 & 1.006 & 0.999 \\
\hline $\mathrm{Ti}$ & 0.000 & 0.000 & 0.000 & 0.000 & 0.000 & 0.000 & 0.000 & 0.000 & 0.000 & 0.000 & 0.000 & 0.000 & 0.000 & 0.000 & 0.000 & 0.000 \\
\hline Al & 0.000 & 0.000 & 0.000 & 0.000 & 0.000 & 0.000 & 0.000 & 0.000 & 0.000 & 0.000 & 0.000 & 0.000 & 0.000 & 0.000 & 0.000 & 0.000 \\
\hline $\mathrm{Cr}$ & 0.000 & 0.000 & 0.000 & 0.000 & 0.000 & 0.000 & 0.001 & 0.000 & 0.000 & 0.000 & 0.000 & 0.000 & 0.000 & 0.001 & 0.000 & 0.000 \\
\hline $\mathrm{Fe}$ & 0.206 & 0.206 & 0.201 & 0.204 & 0.183 & 0.157 & 0.189 & 0.204 & 0.180 & 0.201 & 0.188 & 0.196 & 0.209 & 0.201 & 0.201 & 0.213 \\
\hline Mn & 0.002 & 0.003 & 0.002 & 0.002 & 0.002 & 0.003 & 0.004 & 0.004 & 0.003 & 0.002 & 0.002 & 0.004 & 0.002 & 0.003 & 0.002 & 0.002 \\
\hline Mg & 1.776 & 1.775 & 1.776 & 1.777 & 1.809 & 1.822 & 1.799 & 1.786 & 1.807 & 1.788 & 1.796 & 1.794 & 1.802 & 1.788 & 1.779 & 1.781 \\
\hline $\mathrm{Ca}$ & 0.001 & 0.006 & 0.000 & 0.004 & 0.001 & 0.001 & 0.001 & 0.001 & 0.000 & 0.001 & 0.001 & 0.000 & 0.001 & 0.000 & 0.001 & 0.001 \\
\hline $\mathbf{N i}$ & 0.008 & 0.006 & 0.008 & 0.006 & 0.009 & 0.008 & 0.006 & 0.008 & 0.009 & 0.007 & 0.006 & 0.004 & 0.004 & 0.002 & 0.004 & 0.006 \\
\hline Fo $(\%)$ & 89.51 & 89.46 & 89.72 & 89.62 & 90.72 & 91.92 & 90.31 & 89.59 & 90.83 & 89.77 & 90.41 & 89.97 & 89.50 & 89.74 & 89.73 & 89.25 \\
\hline Fa & 10.38 & 10.37 & 10.16 & 10.26 & 9.15 & 7.94 & 9.49 & 10.22 & 9.02 & 10.10 & 9.49 & 9.85 & 10.41 & 10.10 & 10.16 & 10.66 \\
\hline $\mathrm{Tp}$ & 0.09 & 0.16 & 0.11 & 0.10 & 0.12 & 0.13 & 0.19 & 0.18 & 0.14 & 0.12 & 0.10 & 0.18 & 0.09 & 0.17 & 0.12 & 0.09 \\
\hline Mg\# & 0.90 & 0.90 & 0.90 & 0.90 & 0.91 & 0.92 & 0.90 & 0.90 & 0.91 & 0.90 & 0.90 & 0.90 & 0.90 & 0.90 & 0.90 & 0.89 \\
\hline
\end{tabular}




\subsubsection{Ortho- and Clinopyroxenes}

SEM microscopic observations indicate the presence of numerous pyroxenes in the beach sediments. Analysis of the chemical composition of minerals (Table 7) allowed separating augite, pigeonite, diopside and enstatite. An overwhelming majority of pyroxenes (about 85\%) is composed of enstatite and about $12 \%-15 \%$ of diopside. Augite and pigeonite are quite rare (1\%-2\%). Pyroxenes occur in the $0.1-0.5 \mathrm{~mm}$ grain class. Usually, they form subhedral and anhedral crystals, without visible cleavage. They coexist numerously as a paragenesis with amphibole and ilmenite (Figure 9). A characteristic feature of these minerals is the jagged end of the crystal. Pyroxenes undergo slow serpentinization processes that result in the formation of serpentine minerals with a fibrous structure.

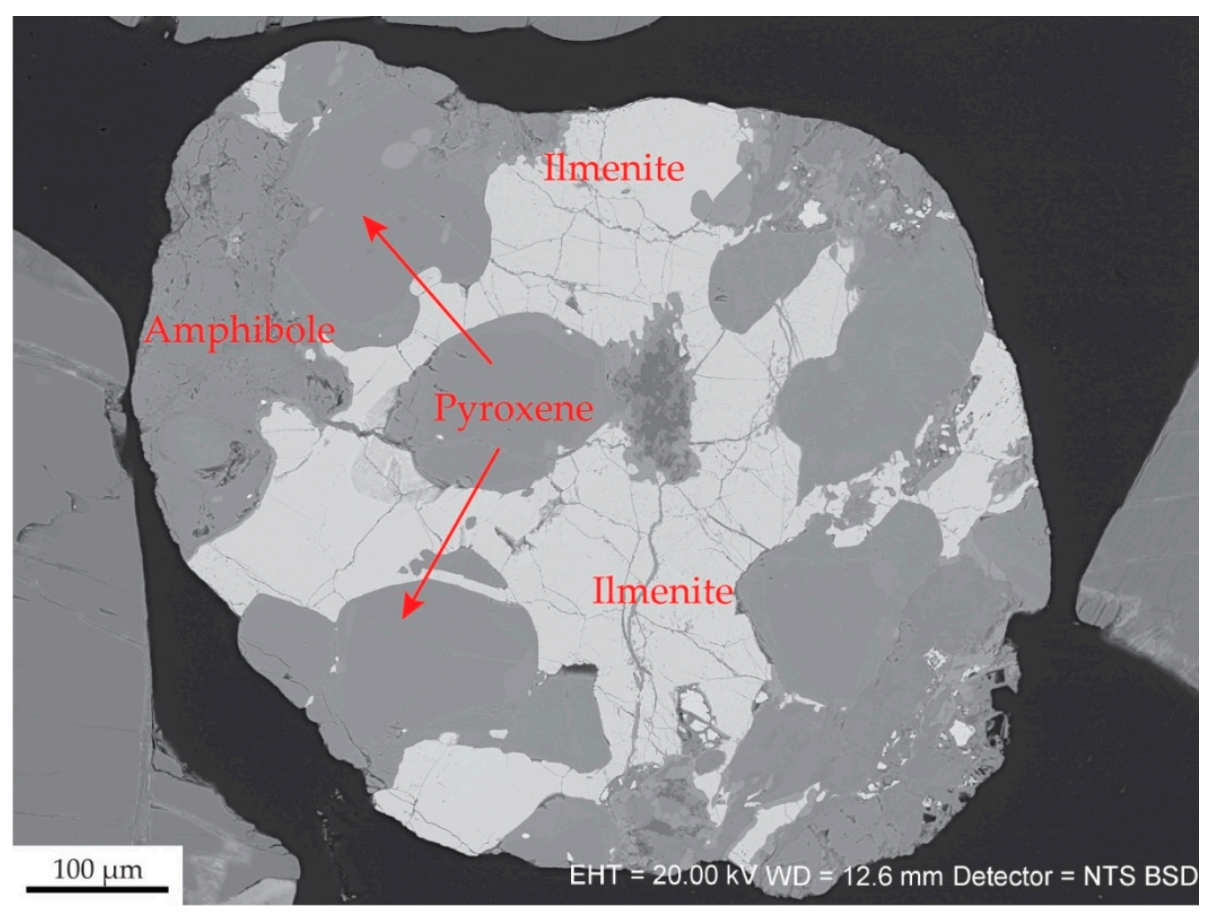

Figure 9. Pyroxene with paragenesis amphibole and ilmenite. SEM-BSE image.

The chemical composition of enstatite $\left(\mathrm{En}_{86.5-90.4} \mathrm{Wo}_{0.1-4.50}\right)$ is typical for minerals from this group, which occur in ultramafic rocks and contain $\mathrm{SiO}_{2}(54.22 \mathrm{wt} \%-57.29 \mathrm{wt} \%)$ and $\mathrm{MgO}(31.51 \mathrm{wt} \%-$ $34.50 \mathrm{wt} \%)$. The content of $\mathrm{Al}_{2} \mathrm{O}_{3}$ is low (0.89 wt $\left.\%-4.23 w \mathrm{t} \%\right)$. Additionally, $\mathrm{NiO}(0.01 \mathrm{wt} \%-0.15 \mathrm{wt} \%)$ and $\mathrm{Cr}_{2} \mathrm{O}_{3}(0.38 \mathrm{wt} \%-0.92 \mathrm{wt} \%)$ are present in small amounts. These metals commonly occur in magnesium-rich orthopyroxenes from ultramafic rocks [51]. The magnesium index of enstatite is $\mathrm{Mg \#}$ $0.90-0.92$.

The chemical composition of diopside $\left(\mathrm{En}_{42.0-48.4} \mathrm{Wo}_{46.8-53.9}\right)$ (Table 7) shows the presence of this mineral that originates from two sources: 1) mafic; and 2) acid rocks. The minerals' composition depends on the source. Mafic diopsides are characterized by higher contents of $\mathrm{CaO}(22.59 \mathrm{wt} \%-$

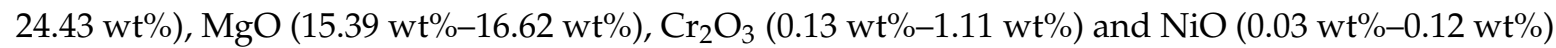
and low contents of $\mathrm{TiO}_{2}(0.03 \mathrm{wt} \%-0.39 \mathrm{wt} \%)$ and $\mathrm{MnO}(0.05 \mathrm{wt} \%-0.20 \mathrm{wt} \%)$ [51], contrary to the diopsides from the acid rocks, consisting of $\mathrm{CaO}(22.29 \mathrm{wt} \%-23.46 \mathrm{wt} \%), \mathrm{MgO}(13.95 \mathrm{wt} \%-15.04 \mathrm{wt} \%)$, $\mathrm{Cr}_{2} \mathrm{O}_{3}$ (up to $0.17 \mathrm{wt} \%$ ), $\mathrm{NiO}$ (up to $0.03 w \mathrm{t} \%$ ), $\mathrm{TiO}_{2}(0.03 \mathrm{wt} \%-0.76 \mathrm{wt} \%$ ) and $\mathrm{MnO}(0.08 w \mathrm{t} \%-$ $0.1 \mathrm{wt} \%$ ) [51]. Diopsides from the acid rocks show high contents of $\mathrm{FeO}$ (up to $5.65 \mathrm{wt} \%$ ) and $\mathrm{Fe}_{2} \mathrm{O}_{3}$ (up to $3.85 \mathrm{wt} \%$ ). The magnesium index $\mathrm{Mg} \#$ for diopside from acid rocks is $0.83-0.86$ and for mafic diopside $\mathrm{Mg} \#$ 0.93-0.97. 
Table 7. Chemical composition of pyroxene in $w \mathrm{t} \%$. EPMA analysis.

\begin{tabular}{|c|c|c|c|c|c|c|c|c|c|c|c|c|c|c|c|c|c|}
\hline & \multicolumn{7}{|c|}{ Enstatite } & \multicolumn{7}{|c|}{ Diopside } & \multirow[t]{2}{*}{ Augite } & \multirow[t]{2}{*}{ Augite } & \multirow[t]{2}{*}{ Pigeonite } \\
\hline & \multicolumn{14}{|c|}{$w t \%$} & & & \\
\hline $\mathrm{SiO}_{2}$ & 55.17 & 56.17 & 57.12 & 55.65 & 55.16 & 57.29 & 56.49 & 50.04 & 50.12 & 52.53 & 51.70 & 51.88 & 51.52 & 50.79 & 51.48 & 51.34 & 54.46 \\
\hline $\mathrm{TiO}_{2}$ & 0.05 & 0.07 & 0.02 & 0.04 & 0.03 & 0.01 & 0.02 & 0.40 & 0.48 & 0.28 & 0.10 & 0.19 & 0.12 & 0.19 & 0.25 & 0.44 & 0.06 \\
\hline $\mathrm{Al}_{2} \mathrm{O}_{3}$ & 4.23 & 2.71 & 1.16 & 2.86 & 3.53 & 1.11 & 2.08 & 3.94 & 3.62 & 1.30 & 4.39 & 4.26 & 3.91 & 5.63 & 6.10 & 1.82 & 4.19 \\
\hline $\mathrm{Cr}_{2} \mathrm{O}_{3}$ & 0.84 & 0.79 & 0.38 & 0.56 & 0.58 & 0.58 & 0.60 & 0.17 & 0.00 & 0.04 & 0.99 & 0.81 & 0.88 & 1.08 & 0.97 & 0.01 & 0.89 \\
\hline $\mathrm{Fe}_{2} \mathrm{O}_{3}$ & 0.00 & 0.00 & 0.00 & 0.00 & 0.12 & 0.00 & 0.00 & 3.66 & 3.85 & 2.51 & 1.13 & 1.27 & 1.51 & 1.63 & 0.93 & 1.91 & 0.44 \\
\hline $\mathrm{FeO}$ & 6.58 & 6.24 & 6.59 & 6.52 & 6.12 & 6.44 & 6.29 & 3.82 & 3.93 & 5.65 & 0.96 & 1.28 & 0.81 & 1.04 & 2.29 & 11.53 & 5.09 \\
\hline $\mathrm{MnO}$ & 0.13 & 0.07 & 0.20 & 0.15 & 0.16 & 0.13 & 0.15 & 0.21 & 0.17 & 0.51 & 0.06 & 0.12 & 0.10 & 0.06 & 0.12 & 0.35 & 0.16 \\
\hline $\mathrm{MgO}$ & 32.78 & 33.49 & 33.99 & 33.38 & 33.32 & 33.47 & 33.62 & 14.76 & 13.95 & 15.04 & 16.18 & 16.25 & 16.62 & 15.44 & 17.48 & 13.38 & 31.62 \\
\hline $\mathrm{CaO}$ & 0.53 & 0.71 & 0.74 & 0.41 & 0.37 & 0.61 & 0.91 & 22.60 & 23.76 & 22.30 & 24.43 & 24.18 & 24.18 & 23.72 & 19.68 & 19.27 & 2.68 \\
\hline $\mathrm{Na}_{2} \mathrm{O}$ & 0.04 & 0.01 & 0.03 & 0.03 & 0.02 & 0.00 & 0.06 & 0.19 & 0.20 & 0.33 & 0.18 & 0.23 & 0.08 & 0.42 & 0.67 & 0.30 & 0.06 \\
\hline $\mathrm{K}_{2} \mathrm{O}$ & 0.00 & 0.00 & 0.00 & 0.00 & 0.00 & 0.00 & 0.00 & 0.00 & 0.00 & 0.00 & 0.00 & 0.00 & 0.00 & 0.01 & 0.00 & 0.00 & 0.00 \\
\hline $\mathrm{NiO}$ & 0.12 & 0.13 & 0.14 & 0.15 & 0.13 & 0.15 & 0.12 & 0.00 & 0.00 & 0.00 & 0.03 & 0.11 & 0.09 & 0.04 & 0.05 & 0.02 & 0.08 \\
\hline \multirow[t]{2}{*}{ Total } & 100.68 & 100.37 & 100.38 & 99.74 & 99.52 & 99.87 & 100.34 & 99.78 & 100.09 & 100.49 & 100.13 & 100.58 & 99.82 & 100.05 & 99.99 & 100.36 & 99.73 \\
\hline & \multicolumn{17}{|c|}{ apfu } \\
\hline $\mathrm{Si}$ & 1.906 & 1.937 & 1.969 & 1.930 & 1.914 & 1.977 & 1.951 & 1.856 & 1.861 & 1.940 & 1.879 & 1.880 & 1.879 & 1.850 & 1.859 & 1.930 & 1.894 \\
\hline $\mathrm{Ti}$ & 0.001 & 0.002 & 0.000 & 0.001 & 0.001 & 0.000 & 0.000 & 0.011 & 0.013 & 0.008 & 0.003 & 0.005 & 0.003 & 0.005 & 0.007 & 0.013 & 0.002 \\
\hline Al & 0.172 & 0.110 & 0.047 & 0.117 & 0.144 & 0.045 & 0.085 & 0.172 & 0.158 & 0.057 & 0.188 & 0.182 & 0.168 & 0.242 & 0.259 & 0.081 & 0.172 \\
\hline $\mathrm{Cr}$ & 0.023 & 0.022 & 0.010 & 0.015 & 0.016 & 0.016 & 0.016 & 0.005 & 0.000 & 0.001 & 0.028 & 0.023 & 0.025 & 0.031 & 0.028 & 0.000 & 0.025 \\
\hline $\mathrm{Fe}^{3+}$ & 0.000 & 0.000 & 0.000 & 0.000 & 0.003 & 0.000 & 0.000 & 0.102 & 0.107 & 0.070 & 0.031 & 0.035 & 0.041 & 0.045 & 0.025 & 0.054 & 0.012 \\
\hline $\mathrm{Fe}^{2+}$ & 0.183 & 0.180 & 0.190 & 0.189 & 0.178 & 0.186 & 0.182 & 0.118 & 0.122 & 0.174 & 0.029 & 0.039 & 0.025 & 0.032 & 0.069 & 0.363 & 0.148 \\
\hline Mn & 0.004 & 0.002 & 0.006 & 0.005 & 0.005 & 0.004 & 0.004 & 0.007 & 0.005 & 0.016 & 0.002 & 0.004 & 0.003 & 0.002 & 0.004 & 0.011 & 0.005 \\
\hline $\mathrm{Mg}$ & 1.688 & 1.722 & 1.747 & 1.726 & 1.724 & 1.773 & 1.730 & 0.816 & 0.772 & 0.828 & 0.876 & 0.878 & 0.904 & 0.838 & 0.941 & 0.750 & 1.640 \\
\hline $\mathrm{Ca}$ & 0.020 & 0.026 & 0.027 & 0.015 & 0.014 & 0.023 & 0.034 & 0.898 & 0.945 & 0.882 & 0.951 & 0.939 & 0.945 & 0.926 & 0.762 & 0.776 & 0.100 \\
\hline $\mathrm{Na}$ & 0.002 & 0.000 & 0.002 & 0.002 & 0.001 & 0.000 & 0.004 & 0.014 & 0.015 & 0.023 & 0.012 & 0.016 & 0.006 & 0.030 & 0.047 & 0.022 & 0.004 \\
\hline $\mathbf{K}$ & 0.000 & 0.000 & 0.000 & 0.000 & 0.000 & 0.000 & 0.000 & 0.000 & 0.000 & 0.000 & 0.000 & 0.000 & 0.000 & 0.000 & 0.000 & 0.000 & 0.000 \\
\hline $\mathrm{Ni}$ & 0.003 & 0.003 & 0.004 & 0.004 & 0.004 & 0.004 & 0.003 & 0.000 & 0.000 & 0.000 & 0.001 & 0.003 & 0.003 & 0.001 & 0.001 & 0.000 & 0.002 \\
\hline Mg\# & 0.90 & 0.91 & 0.90 & 0.90 & 0.90 & 0.91 & 0.91 & 0.87 & 0.86 & 0.83 & 0.97 & 0.96 & 0.97 & 0.96 & 0.93 & 0.67 & 0.92 \\
\hline En * & 0.89 & 0.89 & 0.89 & 0.89 & 0.90 & 0.90 & 0.89 & 0.46 & 0.42 & 0.44 & 0.47 & 0.47 & 0.48 & 0.47 & 0.53 & 0.40 & 0.87 \\
\hline Fs * & 0.10 & 0.09 & 0.10 & 0.09 & 0.09 & 0.09 & 0.09 & 0.07 & 0.07 & 0.09 & 0.02 & 0.02 & 0.01 & 0.02 & 0.04 & 0.19 & 0.08 \\
\hline Wo * & 0.01 & 0.01 & 0.01 & 0.01 & 0.01 & 0.01 & 0.01 & 0.49 & 0.51 & 0.47 & 0.51 & 0.51 & 0.50 & 0.52 & 0.43 & 0.41 & 0.05 \\
\hline
\end{tabular}

* end-member were calculated. 


\section{Discussion}

\subsection{Provenance Area}

The collision of the Indo-Australian and Euro-Asian plates, formation of numerous subduction zones and related extension processes led to a series of changes on the regional level in SE Asia, including New Guinea Island [38,39,52]. Cenozoic tectonic collisions with island-arc terranes (Tosem, Amanab, Weyland, Gauttier, Cyclops) and closing of marginal basins led to the formation of numerous ophiolites in a supra-subduction environment $[46,47,53]$. The creation of the New Guinea ophiolites was associated with the formation of two unevenly-aged marginal basins in the zone surrounding the Indo-Australian Plate [52,53]. The development of the basins was determined by convergence processes between the Indo-Australian Plate (which was at first moving to the north-east and then to the north) and smaller oceanic plates: the Philippine Sea Plate and the Caroline Plate [39].

Ophiolite may represent a wide variety of lithological construction and geochemical development. Chromian spinels may be formed in depleted mantle section of ophiolites formed in supra-subduction zone (SSZ) environments due to partial melting and melt-rock reaction. Mantle sequences of many ophiolites contain podiform chromitites composed of magnesiochromite \pm olivine \pm other silicate phases $[46,54,55]$.

In the Indonesian part of New Guinea Island, five zones with podiform-type ophiolite sequences were localized (Figure 1). Potentially, they could be a source of chromium and cobalt [15]. In the Waropen Regency, no similar deposit structures have been found until now. The Regency area is one of the least geologically known areas of New Guinea. It is due to the morphology of the terrain (strongly hilly area), lack of road infrastructure and rich and dense vegetation which makes it difficult to access particular locations.

The composition of contemporary terrigenous sediment is a result of mutual interactions between the parent rocks and factors acting during the sedimentary cycle [56-58]. The beach sediments from the Waropen Regency have polymineralic composition, which indicates a relatively mixed source area. The contents of heavy minerals in the samples reach $20 \%$, showing high concentration of $\mathrm{Cr}_{2} \mathrm{O}_{3}$ up to $1.17 \mathrm{wt} \%$ and $\mathrm{Ni}$ up to $556 \mathrm{ppm}$. The morphology analysis of heavy mineral grains indicates two generations of different roundness: 1) moderate and well rounded, discoidal and spherical grains - amphiboles, pyroxenes, olivine, andalusite; and 2) poorly rounded, automorphic and elongated grains - chromite, magnetite, rutile and monazite. The spatial distribution and associated grain size and shape depends on the transport, distance, deposition conditions and the physical and chemical properties of minerals. As it is pointed out by Reference [56], olivine, pyroxenes and amphiboles are the least stable minerals, while ilmenite, rutile and chromite - potential indicator minerals - are more physically and chemically stable. The resistant nature of these minerals explains their accumulation in the coastal environment following long-term erosion of the parent rock [59].

Field and satellite analysis of the land surface structure (Figure 1), course and watercourses layout and water sections, indicate that rocks building the western part of the Van-Rees Mountains are the potential source of heavy minerals. The slope gradient direction in these mountains is NNW, similar to the direction towards which permanent watercourses are draining. The New Guinea rivers transport $1.7 \times 10^{9} \mathrm{t}^{-1}$ of sediments into the World Ocean yearly [60]. The amount of incoming mass is spatially differentiated throughout the island. The rivers draining the northern and north-western part of the island are the source of sediments containing quartz, clay minerals and heavy minerals [60].

Fragments of metamorphic rocks are present in the beach sand sediments, mainly chlorite-, quartz- and mica-shales. Additionally, in the Botawa River, alluvial fragments of ultramafic rocks are present. The mineral composition of beach sediments from the Waropen Regency is analogous to the clastic material from the Jayapura region, New Guinea [61]. The original source of contemporary terrigenous sediments in the Jayapura Regency are rocks form the Cyclops Mountains terrane-a core composed of calcium-alkaline metamorphic rocks and an ophiolite sequence which was obducing on top. It is possible that the geologically poorly explored Van-Rees Mountains are part of a terrane or an 
accretionary wedge, unknown until now. According to Reference [34], the Van-Rees Mountains can form an element of a paleo-subduction complex (accretionary prism), separating the North Basin (into the fore-arc basin and back-arc basin) where chromite was generated under supra-subduction conditions.

\subsection{Source of Indicator Minerals}

Based on chromite chemical composition, a range of correlation was determined, which may help in establishing the character of parent rocks and the geotectonic environment. The content of $\mathrm{TiO}_{2}$ is a key indicator for the understanding of the geotectonic environment [62]. In spinels originated from dunites, $\mathrm{TiO}_{2}$ contents are very low and amount to an average of $0.11 \mathrm{wt} \%$, for harzburgites $-0.05 \mathrm{wt} \%$ and lherzholites- $0.06 \mathrm{wt} \%$. The source for chromite is most likely peridotites (Figures 10 and 11A,B) from the Earth's mantle zone, an ophiolite sequence formed under supra-subduction conditions (Figure 11B). Chromian spinels that formed in the initial phase the fore-arc basin creation have geochemical indicators of boninites, while chromites that formed in the back-arc basin show a geochemical composition similar to MORB [63]. The $\mathrm{Al}_{2} \mathrm{O}_{3}$ contents in spinels and pyroxenes are sensitive to the degree of melting rocks from the Earth's mantle. Systematic $\mathrm{Al}_{2} \mathrm{O}_{3}$ decrease in these minerals indicates an increase in peridotite impoverishment [64]. A dependence diagram between $\mathrm{TiO}_{2} \mathrm{wt} \%$ and $\mathrm{Al}_{2} \mathrm{O}_{3} \mathrm{wt} \%$ [62] reveals similarity/affinity of high-Cr spinels generated from the Ti- and Al-depleted magma in the fore-arc basin. Spinels enriched in aluminum, which are genetically related to the back-arc basin, show a geochemically different character. The $\mathrm{Cr} \#$ versus $\mathrm{TiO}_{2} \mathrm{wt} \%$ dependence diagram (Figure 12) indicates that chromite originates from impoverishment peridotites with a slight deviation towards MORB for $\mathrm{Al}$ chromite. The $\mathrm{Cr} / \mathrm{Fe}$ ratio is in the range of $3.40-6.11$, on average 4.45 . The $\mathrm{Cr} / \mathrm{Fe}$ ratio is less than 2.5 in most stratiform chromite ores but is high (2.5-4.5) in ophiolite chromite [16].

The EPMA studies of olivine have shown that it is represented by magnesium varieties with a high magnesium index (Mg\# 90-92). High Mg\# and very low $\mathrm{NiO}$ contents $(0.29 \mathrm{wt} \%-0.45 \mathrm{wt} \%)$ show its geochemical similarity to olivine from dunites and harzburgites of the Cyclops Mountains [45,61]. A similar relationship can be observed in pyroxenes, where the chemical composition is similar to that of pyroxenes from ultramafic rocks. The enstatite chemical composition is typical for orthopyroxene from ultramafic rocks. Enstatite show a low content of $\mathrm{Al}_{2} \mathrm{O}_{3} \quad 0.89 \%-3.03 \%$ and have admixtures of nickel ( $\mathrm{NiO} 0.01 \mathrm{wt} \%-0.15 \mathrm{wt} \%)$ and chromium $\left(\mathrm{Cr}_{2} \mathrm{O}_{3} 0.38 \mathrm{wt} \%-0.92 \mathrm{wt} \%\right)$. The magnesium index (Mg\#) positively correlates with the increasing impoverishment of peridotites. The chemical analysis of the samples indicates that orthopyroxenes are associated with abyssal peridotites (ABP) and the supra-subduction zone in fore-arc basin (diagram according to [67], Figure 13).

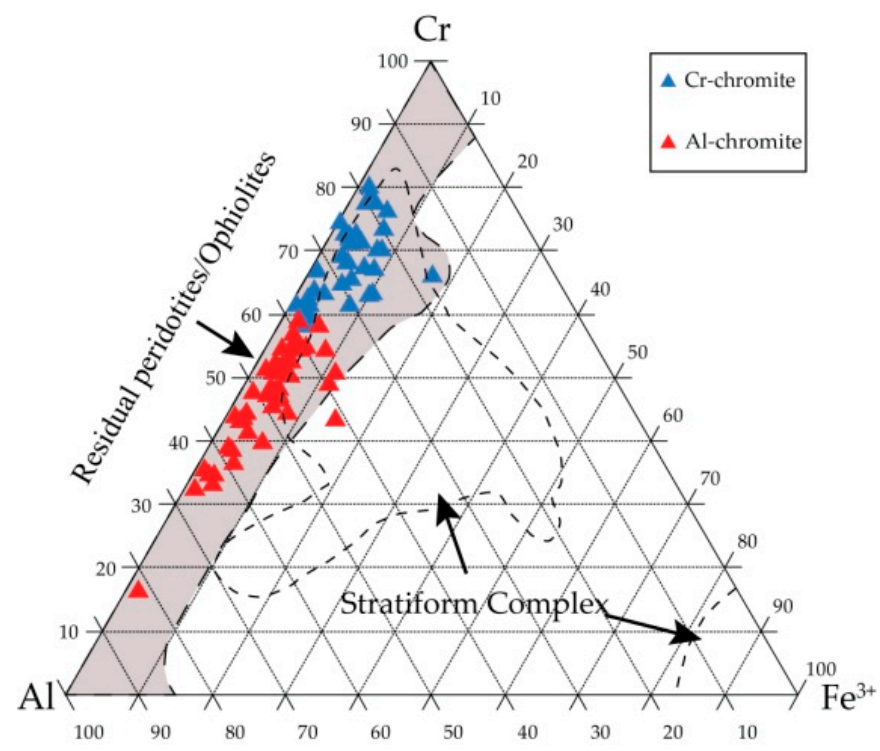

Figure 10. $\mathrm{Cr}-\mathrm{Al}-\mathrm{Fe}^{3+}$ triangle plot of chromite from beach sediments of the Waropen Regency (after [65]). 

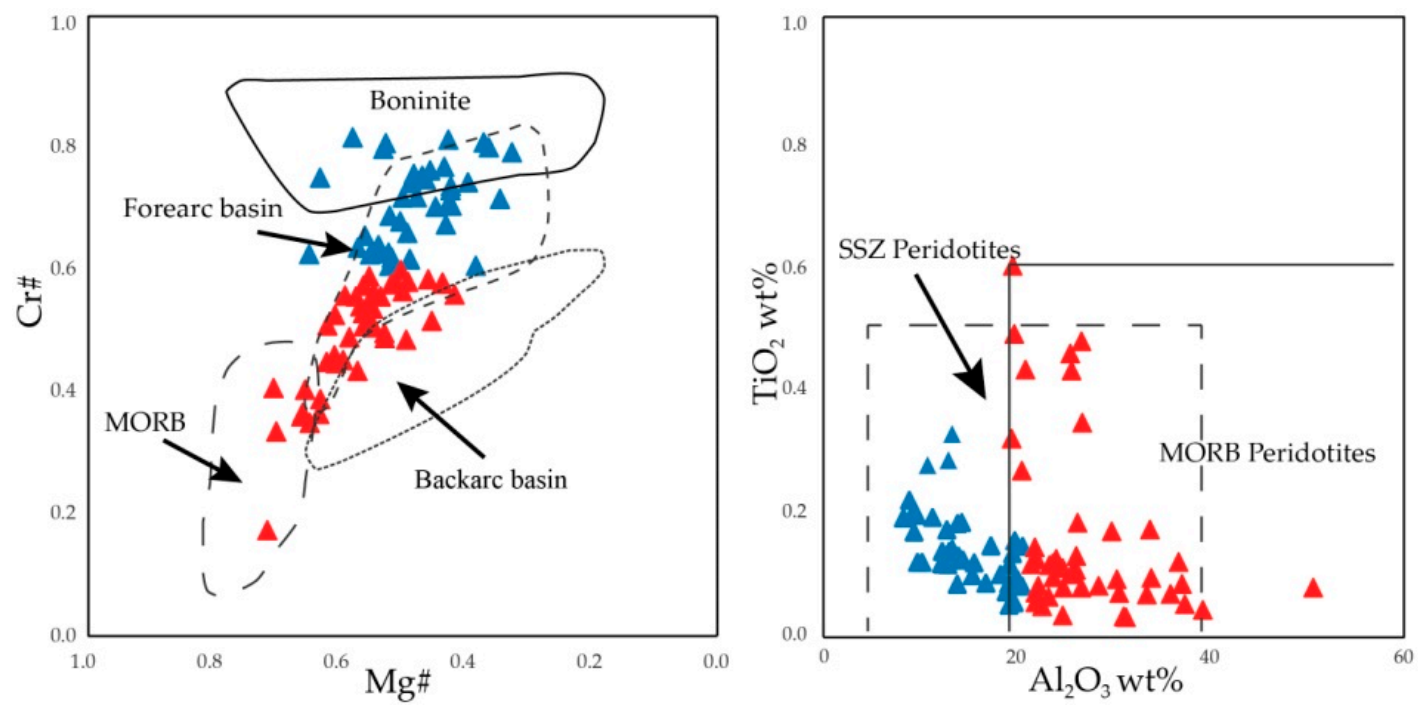

Figure 11. Compositional variations of chromian spinels from the Waropen Regency: (A- on the left

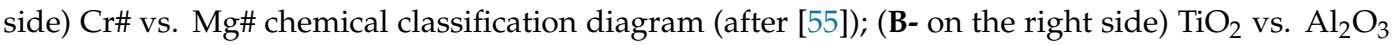
diagram (after [62]).

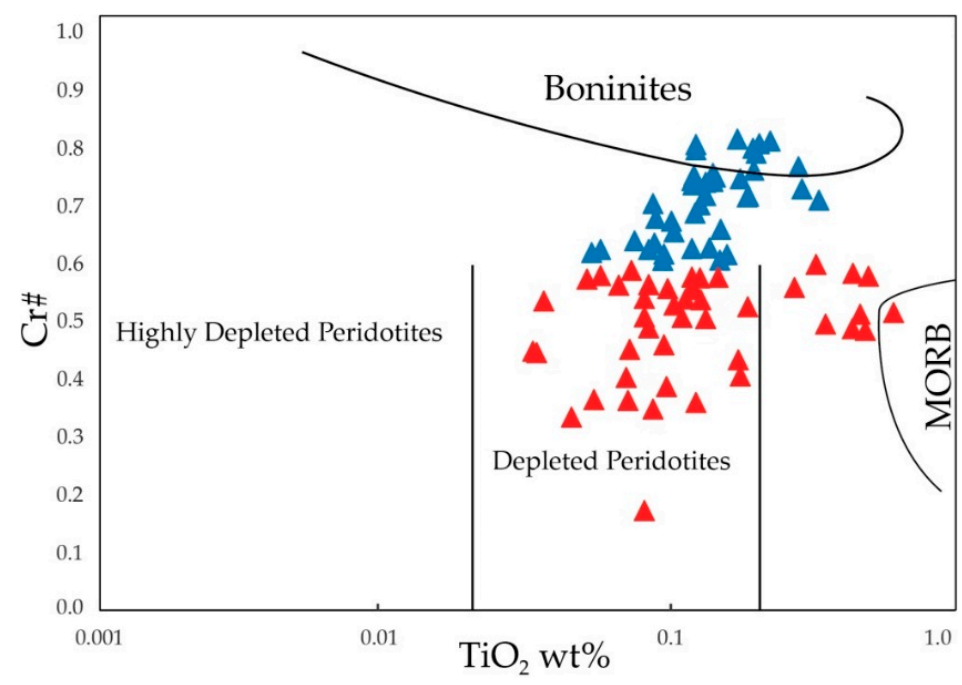

Figure 12. $\mathrm{TiO}_{2}$ versus $\mathrm{Cr} \#$ for chromian spinel (after $\left.[49,55,66]\right)$.
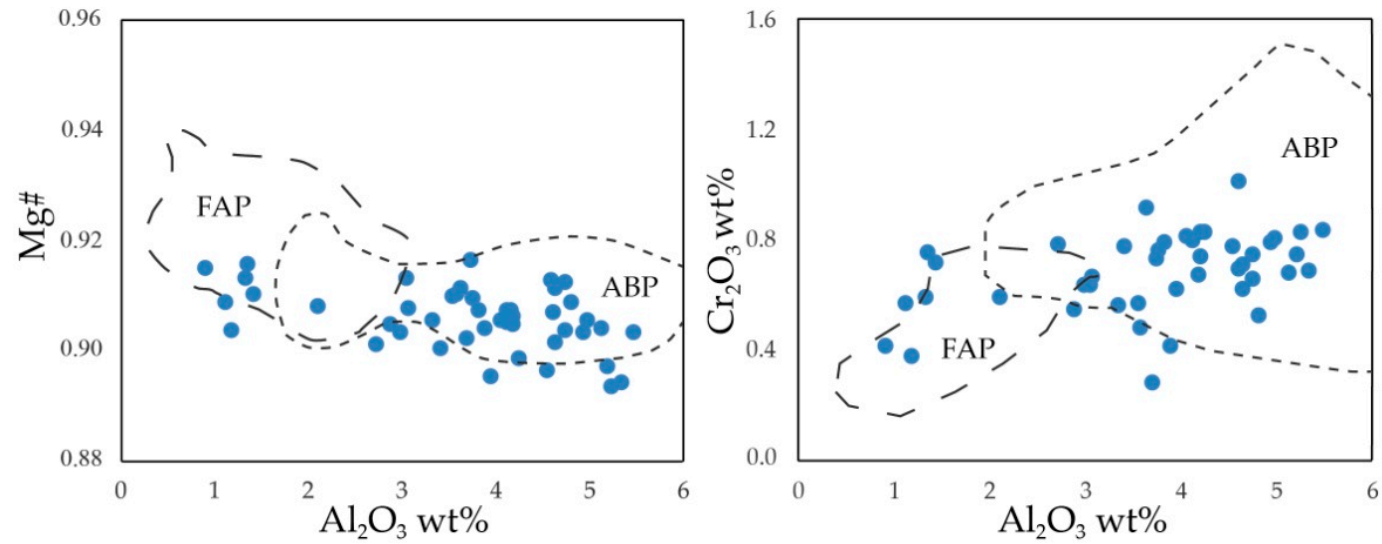

Figure 13. The composition of orthopyroxene: ABP—abyssal peridotites, FAP-fore-arc peridotites (after [67]). 


\subsection{Parental Magma}

The origin of chromian spinels is widely debated within the scientific community $[58,68-74]$. It is generally accepted that their chemical composition depends on the chemical composition of parental magma $[55,62,75]$ and their interaction (melt/rock or melt/melt), previously depleted inside the Earth's mantle in the SSZ zone. The high-Cr spinel $(\mathrm{Cr} \#>60)$ forms through the interaction between magnesium-rich ultramafic magma (boninite composition) [76,77] and variably refractory peridotites [54]. Meanwhile, Al spinels (Cr\# < 60) form from MORB-type tholeiitic magma.

High-Cr spinels from the Waropen Regency were most probably formed in the initial stage of the subduction process from chromium-oversaturated boninite magma, gradually changing their geochemical character towards MORB. The modification of chemical composition of $\mathrm{Cr} \#$ and $\mathrm{Mg \#}$ spinels may reflect a partial melting of original/primary peridotites, magma generation conditions in rock/melt reaction and the metasomatic process impact occurring in the mantle wedge [78,79].

The chemical composition of chromian spinels recreates the composition of an initial melt. Using the regression equation $[62,79,80]$ the $\mathrm{Al}_{2} \mathrm{O}_{3}$ and $\mathrm{TiO}_{2}$ ( $\mathrm{wt} \%$ ) value in $\mathrm{Cr}$-spinels was calculated: $\left(\mathrm{Al}_{2} \mathrm{O}_{3 \text { melt }}\right)=5.2253 \ln \left(\mathrm{Al}_{2} \mathrm{O}_{3 \text { spinel }}\right)-1.232 ;\left(\mathrm{TiO}_{2 \text { melt }}\right)=1.0897 \ln \left(\mathrm{TiO}_{2 \text { spinel }}\right)+0.0892$;

for Al-spinels from MORB:

$$
\left(\mathrm{Al}_{2} \mathrm{O}_{3 \text { melt }}\right)=4.1386 \ln \left(\mathrm{Al}_{2} \mathrm{O}_{3 \text { spinel }}\right)+2.2828 ;\left(\mathrm{TiO}_{2 \text { melt }}\right)=0.708 \ln \left(\mathrm{TiO}_{2 \text { spinel }}\right)+1.6436
$$

To calculate $\mathrm{FeO} / \mathrm{MgO}$ melt [81], empirical formula was used:

$\ln (\mathrm{FeO} / \mathrm{MgO})_{\text {spinel }}=0.47-1.07 \mathrm{Al} \#_{\text {spinel }}+0.64 \mathrm{Fe}_{\text {spinel }}+\ln (\mathrm{FeO} / \mathrm{MgO})_{\text {liquid }}$, where $\mathrm{MgO}$ and $\mathrm{FeO}$ are in $\mathrm{wt}_{\mathrm{t}} \%$. Initial melt calculation (Table 5) shows that $\mathrm{Cr}$ spinels originate from boninite magma, which was characterized by the contents of $\mathrm{Al}_{2} \mathrm{O}_{3 \text { melt }}(9.91 \mathrm{wt} \%-14.69 \mathrm{wt} \%), \mathrm{TiO}_{2 \text { melt }}(0.15 \mathrm{wt} \%-$ $0.54 \mathrm{wt} \%)$ and $\mathrm{FeO} / \mathrm{MgO}_{\text {melt }}(0.17 \mathrm{wt} \%-0.99 \mathrm{wt} \%)$. Aluminum spinels have a different $\mathrm{TiO}_{2}$ content, which may result from a partial enrichment of parental magma $\mathrm{TiO}_{2 \text { melt }}(0.01 \mathrm{wt} \%-1.29 \mathrm{wt} \%)$. This phenomenon (Figure 14) is manifested by the trend change of boninite melt into MORB, which may be the result of a melt/melt interaction or reaction between different melt with parent rock $[78,79]$.

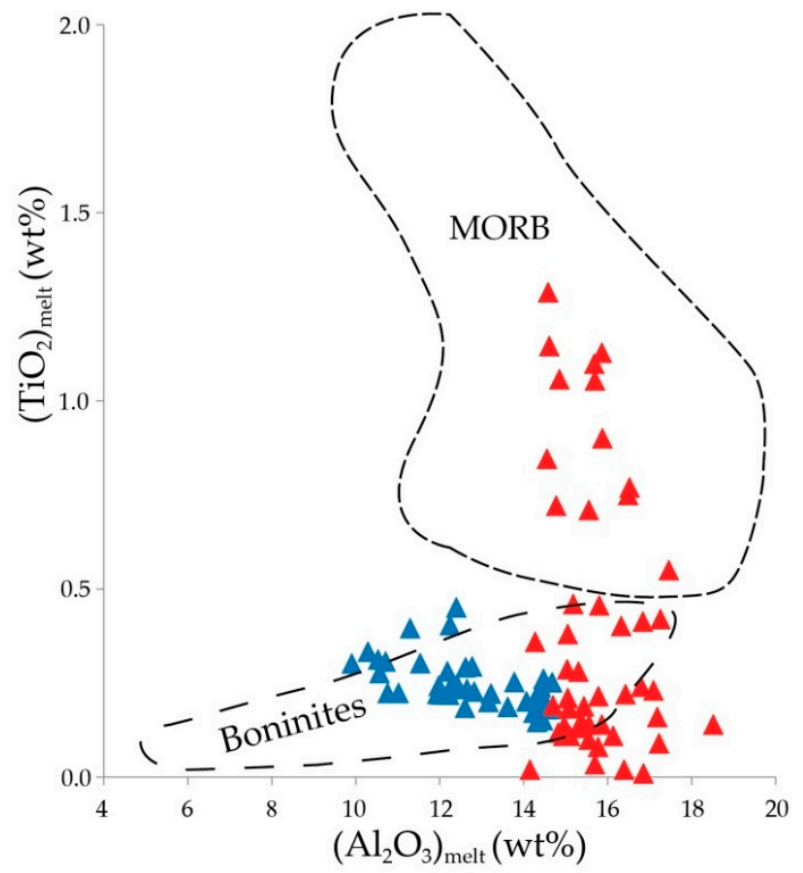

Figure 14. $\mathrm{Al}_{2} \mathrm{O}_{3 \text { melt }} \mathrm{vs}$. $\mathrm{TiO}_{2 \text { melt }}$ of the melt calculated as in equilibrium with chromite from samples. Fields for boninites and MORB (Mid Ocean Ridge Basalts) (after [82]). 


\subsection{Further Exploration and Research Destination}

The geological prospection enables to identify the most promising area for mineral exploration. The examinations provided evidence for abnormally high concentrations of useful metals in the river samples- $\mathrm{Cr}_{2} \mathrm{O}_{3}$ up to $24.83 \mathrm{wt} \%$ and $\mathrm{Au} 3.05 \mathrm{ppm}$. With current knowledge, it is impossible to adopt reliable parameters of minimum average and marginal contents of useful components in the deposit (cut off). Based on the obtained results, it can be assumed that the parent rocks of metals are the rocks from the western part of the Van Rees Mountains complex. It was determined that the beach sediments are not promising as mineral deposits and further work should be focused on analysis of fluvial sediments. Future research should concentrate on identification of useful minerals and their technological properties, as well as on abundance of mineral resources. The Botawa River sediments show a high concentration of $\mathrm{Cr}_{2} \mathrm{O}_{3}$. Therefore, it is likely that further exploration areas located in the upstream part of the Botawa River will contain even higher amounts of chromian spinels. It may lead to the discovery of rich chromium-bearing placer deposits.

The chemical composition analysis of chromian spinels (Table 5) has allowed establishing the preliminary character and source of chromium-bearing rocks that were generated from initial rock melt from magma composed of boninite and MORB. According to contemporary geodynamic models of New Guinea $[45,47,83]$, the northern surrounding zone of the Indo-Australian Plate was a part of the active edge, along with the subduction zone. As a result of structural changes, terranes were accreted and the ophiolites generation took place in the SSZ environment $[45,53]$.

\section{Conclusions}

Geological prospection of the Botawa River sediments and beach sediments has demonstrated the presence of indicator minerals, such as chromite, magnesium olivine and pyroxenes, characteristic for chromium deposits of podiform type. The content of $\mathrm{Cr}_{2} \mathrm{O}_{3}$ increases linearly towards the potential parent rock source- the Van Rees Mountains. The concentration of $\mathrm{Cr}_{2} \mathrm{O}_{3}$ in beach sediments is up to $1.17 \mathrm{wt} \%$, while in heavy minerals concentrate from the Botawa River it increases to $24.83 \mathrm{wt} \%$. The Van Rees Massif may form a previously unknown terrane, which is linked structurally and geochemically to the Cyclops Mountains complex in the Jayapura region (New Guinea). It is certified by chemical analyses of chromium spinels, pyroxenes, olivines and the presence of fragments of metamorphic rocks-mica and chlorites shales. Detailed geochemical research of the complex will allow finding its more specific geotectonic position.

The chemical analysis of chromian spinels indicates their origin from depleted peridotites, formed in back-arc and fore-arc basins under supra-subduction conditions. Chromian spinels form a continuous spinel-magnesiochromite-chromite series. The analysis of pyroxenes indicates that their grains originate from ultramafic rocks. Chromium spinels need further detailed analysis to establish the conditions of melt generation, its changeability and the possibility of potential concentration of metals from the Pt group.

High concentration of $\mathrm{Cr}_{2} \mathrm{O}_{3}$ in the alluvial sediments may be a clue to finding a new potential source of chromite in New Guinea Island. To confirm this statement, it is necessary to conduct further detailed studies in the upstream Botawa River valley.

Author Contributions: Conceptualization, K.Z., K.S. and A.P.; methodology, K.Z.; software, K.Z.; validation, K.S., K.Z. and A.P.; formal analysis, K.Z.; investigation, K.Z., P.K. and A.P.; resources, A.P.; data curation, K.Z. and K.S.; writing - original draft preparation, K.Z.; writing - review and editing, K.Z. and K.S.; visualization, K.Z.; supervision, A.P.; project administration, K.Z., K.S. and A.P.; funding acquisition, K.S. and K.Z. All authors have read and agreed to the published version of the manuscript.

Funding: This research was supported by statutory funds from Polish Geological Institute—National Research Institute and Warsaw University.

Acknowledgments: Authors would like to express their gratitude towards the reviewers for their useful remarks and comments which allowed the authors to improve this paper.

Conflicts of Interest: The authors declare no conflict of interest. 


\section{References}

1. McClenaghan, M.B.; Plouffe, A.; Paulen, R.C.; Houlé, M.; Jackson, S.E.; Peter, J.M. Overview of Indicator Mineral Research at the Geological Survey of Canada-An Update. Explore 2016, 170, 4-15.

2. McClenaghan, M.B. Indicator Mineral Methods in Mineral Exploration. Geochem. Explor. Environ. Anal. 2005, 5, 233-245. [CrossRef]

3. Morton, A.C.; Hallsworth, C. Stability of Detrital Heavy Minerals During Burial Diagenesis. In Developments in Sedimentology; Mange, M.A., Wright, D.T., Eds.; Elsevier Science: Amsterdam, The Netherlands, 2007; Volume 58, pp. 215-240. [CrossRef]

4. Pisiak, L.K.; Canil, D.; Lacourse, T.; Plouffe, A.; Ferbey, T. Magnetite as an Indicator Mineral in the Exploration of Porphyry Deposits: A Case Study in Till Near the Mount Polley Cu-Au Deposit, British Columbia, Canada. Econ. Geol. 2017. [CrossRef]

5. Cooke, D.R.; Agnew, P.; Hollings, P.; Baker, M.; Chang, Z.; Wilkinson, J.J.; White, N.C.; Zhang, L.; Thompson, J.; Fox, N.; et al. Porphyry Indicator Minerals (PIMS) and Porphyry Vectoring and Fertility Tools (PVFTS)-Indicators of Mineralization Styles and Recorders of Hypogene Geochemical Dispersion Halos. In Proceedings of the Exploration 17, Sixth Decennial International Conference on Mineral Exploration, Toronto, Canada, 27 July 2017; pp. 457-470.

6. Savel'Eva, G.N.; Batanova, V.G.; Sobolev, A.V.; Kuz'Min, D.V. Minerals of Mantle Peridotites: Indicators of Chromium Ores in Ophiolites. Dokl. Earth Sci. 2013, 452, 963-966. [CrossRef]

7. McClenaghan, M.B.; Cabri, L.J. Review of Gold and Platinum Group Element (PGE) Indicator Minerals Methods for Surficial Sediment Sampling. Geochem. Explor. Environ. Anal. 2011, 11, 251-263. [CrossRef]

8. Zhmodik, S.M.; Nesterenko, G.V.; Airiyants, E.V.; Belyanin, D.K.; Kolpakov, V.V.; Podlipsky, M.Y.; Karmanov, N.S. Alluvial Platinum-Group Minerals as Indicators of Primary PGE Mineralization (Placers of Southern Siberia). Russ. Geol. Geophys. 2016, 50, 524-534. [CrossRef]

9. Stendal, H.; Theobald, P.K. Heavy-Mineral Concentrates in Geochemical Exploration. In Handbook of Exploration Geochemistry; Hale, M., Plant, J.A., Eds.; Elsevier Science: Amsterdam, The Netherlands, 1994; Volume 6, pp. 185-225. [CrossRef]

10. Gurney, J.J. A Correlation between Garnets and Diamonds in Kimberlites. In Kimberlite Occurrence and Origin: A Basis for Conceptual Models in Exploration 8; Geology Department and University Extension, University of Western Australia: Perth, Australia, 1984; pp. 143-166.

11. Zozulya, D.R.; Peltonen, P.; O'Brien, H. Pyrope and Cr-Diopside as Indicators of Mantle Structure and Diamond Depth Facies in the Kola Region. Geol. Ore Depos. 2008, 50, 524-534. [CrossRef]

12. Plant, J.A.; Hale, M.; Ridgeway, J. Regional Geochemistry Based on Stream Sediment Sampling. In Exploration 87 Proceeding AEG symp; Ontario Geological Survey: Toronto, ON, Canada, 1989; Volume 3, pp. 384-404.

13. Hall, R. Indonesia, Geology. In Encyclopedia of Islands; Gillespie, R.C.D., Clauge, D., Eds.; University of California Press: Berkeley, CA, USA, 2009; pp. 454-460.

14. Katili, J.A. Volcanism and Plate Tectonics in the Indonesian Island Arcs. Tectonophysics 1975, 26, 165-188. [CrossRef]

15. Ernowo, O.P. Review of Chromite Deposits of Indonesia. Bul. Sumber Daya Geol. 2010, 5, 10-19.

16. Stowe, C.W.S. Compositions and tectonic settings of chromite deposits through time. Econ. Geol. 1994, 89, 528-546. [CrossRef]

17. Cameron, E.N. Evolution of the lower critical zone, central sector, eastern bushveld complex and its chromite deposits. Econ. Geol. 1980, 75, 845-871. [CrossRef]

18. Eales, H.V.; Botha, W.J.; Hattingh, P.J.; De Klerk, W.J.; Maier, W.D.; Odgers, A.T.R. The mafic rocks of the Bushveld complex: A review of emplacement and crystallization history and mineralization, in the light of recent data. J. African Earth Sci. 1993, 16, 121-142. [CrossRef]

19. Alapieti, T.T.; Kujanpaa, J.; Lahtinen, J.J.; Papunen, H. The Kemi stratiform chromitite deposit, northern Finland. Econ. Geol. 1989, 84, 1057-1077. [CrossRef]

20. Alapieti, T.T.; Filén, B.A.; Lahtinen, J.J.; Lavrov, M.M.; Smolkin, V.F.; Voitsekhovsky, S.N. Early Proterozoic layered intrusions in the northeastern part of the Fennoscandian Shield. Mineral. Petrol. 1990, 42, 1-22. [CrossRef] 
21. Higgins, S.J.; Snyder, G.A.; Mitchell, J.N.; Taylor, L.A.; Sharkov, E.V.; Bogatikov, O.A.; Grokhovskaya, T.L.; Chistyakov, A.V.; Ganin, V.A.; Grinevich, N.G. Petrology of the Early Proterozoic Burakovsky layered intrusion, southern Karelia, Russia: Mineral and whole-rock major-element chemistry. Can. J. Earth Sci. 1997, 34, 390-406. [CrossRef]

22. Sharkov, E.V.; Bogatikov, O.A.; Grokhovskaya, T.L.; Chistyakov, A.V.; Ganin, V.A.; Grinevich, N.G.; Snyder, G.A.; Taylor, L.A. Petrology and Ni-Cu-Cr-PGE mineralization of the largest mafic pluton in europe: The early proterozoic burakovsky layered intrusion, Karelia, Russia. Int. Geol. Rev. 1995, 37, 509-525. [CrossRef]

23. Beqiraj, A.; Masi, U.; Violo, M. Geochemical characterization of podiform chromite ores from the ultramafic massif of Bulqiza (Eastern Ophiolitic Belt, Albania) and hints for exploration. Explor. Min. Geol. 2000, 9, 149-156. [CrossRef]

24. Uysal, I.; Tarkian, M.; Sadiklar, M.B.; Zaccarini, F.; Meisel, T.; Garuti, G.; Heidrich, S. Petrology of Al- and Cr-Rich Ophiolitic Chromitites from the Muğla, SW Turkey: Implications from composition of chromite, solid inclusions of platinum-group mineral, silicate and base-metal mineral and Os-isotope geochemistry. Contrib. Mineral. Petrol. 2009, 158, 659-674. [CrossRef]

25. Uysal, I.; Sadiklar, M.B.; Tarkian, M.; Karsli, O.; Aydin, F. Mineralogy and Composition of the Chromitites and their Platinum-Group Minerals from Ortaca (Muğla-SW Turkey): Evidence for ophiolitic chromitite genesis. Mineral. Petrol. 2005, 83, 219-242. [CrossRef]

26. Hock, M.; Friedrich, G.; Plüger, W.L.; Wichowski, A. Refractory- and Metallurgical-Type Chromite Ores, Zambales Ophiolite, Luzon, Philippines. Miner. Depos. 1986, 21, 190-199. [CrossRef]

27. Yumul, G.P.; Balce, G.R.; Dimalanta, C.B.; Datuin, R.T. Distribution, Geochemistry and Mineralization Potentials of Philippine Ophiolite and Ophiolitic Sequences. Ofioliti 1997, 22, 47-56.

28. Yumul, G.P.; Balce, G.R. Supra-Subduction Zone Ophiolites As Favorable Hosts For Chromitite, Platinum And Massive Sulfide Deposits. J. Southeast. Asian Earth Sci. 1994, 10, 65-79. [CrossRef]

29. Mosier, D.L.; Singer, D.A.; Moring, B.C.; Galloway, J.P. Podiform Chromite Deposits—Database and Grade and Tonnage Models; U.S. Geological Survey Scientific Investigations Report 5157: Reston, VA, USA, 2012; pp. 1-54.

30. Allen, J.E. Geological investigation of the chromite deposits of California. Calif. J. Mines Geol. 1941, 37, 101-167.

31. Pigram, C.J.; Davies, H.L. Terranes and the accretion history of the New Guinea orogen. BMR J. Aust. Geol. Geophys. 1987, 10, 193-211.

32. Szamałek, K.; Konopka, G.; Zglinicki, K.; Marciniak-Maliszewska, B. New potential source of rare earth elements. Gospod. Surowcami Miner. Miner. Resour. Manag. 2013, 29, 59-76. [CrossRef]

33. Dow, D.B.; Hartono, U. The Nature of the Crust Underlying Cenderawasih (Geelvink) Bay, Irian Jaya. Proc. 11th Ann. Conv. Indon. Petrol. Assoc. 1982, 203-210. [CrossRef]

34. McAdoo, R.L.; Haebig, J.C. Tectonic Elements of the North Irian Basin. Proc. 27th Ann. Conv. Indon. Petrol. Assoc. 1999, 545-562. [CrossRef]

35. Babault, J.; Viaplana-Muzas, M.; Legrand, X.; Van Den Driessche, J.; González-Quijano, M.; Mudd, S.M. Source-to-Sink Constraints on Tectonic and Sedimentary Evolution of the Western Central Range and Cenderawasih Bay (Indonesia). J. Asian Earth Sci. 2018, 156, 265-287. [CrossRef]

36. Blott, S.J.; Pye, K. Gradistat: A Grain Size Distribution and Statistics Package for the Analysis of Unconsolidated Sediments. Earth Surf. Process. Landforms 2001, 26, 1237-1248. [CrossRef]

37. Folk, R.L.; Ward, W.C. Brazos River bar [Texas]; a study in the significance of grain size parameters. J. Sediment. Res. 1957, 27, 3-26. [CrossRef]

38. Hall, R. Cenozoic Geological and Plate Tectonic Evolution of SE Asia and the SW Pacific: Computer-Based Reconstructions, Model and Animations. J. Asian Earth Sci. 2002, 20, 353-434. [CrossRef]

39. Gaina, C.; Müller, D. Cenozoic Tectonic and Depth/Age Evolution of the Indonesian Gateway and Associated Back-Arc Basins. Earth-Science Rev. 2007, 83, 177-203. [CrossRef]

40. Baldwin, S.L.; Fitzgerald, P.G.; Webb, L.E. Tectonics of the New Guinea Region. Annu. Rev. Earth Planet. Sci. 2012, 40, 495-520. [CrossRef]

41. Cullen, A.B.; Pigott, J.D. Post-Jurassic Tectonic Evolution of Papua New Guinea. Tectonophysics 1989, 162, 291-302. [CrossRef]

42. Jaques, A.L.; Robinson, G.P. The Continent/Island-Arc Collision in Northern Papua New Guinea. BMR J. Aust. Geol. Geophys. 1977, 2, 289-303. 
43. Davies, H.L. The Geology of New Guinea-The Cordilleran Margin of the Australian Continent. Episodes 2012, 35, 87-102. [CrossRef]

44. Hamilton, W. Tectonics of the Indonesian Region; US Government Printing Office: Washington, DC, USA, 1979.

45. Monnier, C.; Girardeau, J.; Pubellier, M.; Polvé, M.; Permana, H.; Bellon, H. Petrology and Geochemistry of the Cyclops Ophiolites (Irian Jaya, East Indonesia): Consequences for the Cenozoic Evolution of the North Australian Margin. Mineral. Petrol. 1999, 65, 1-28. [CrossRef]

46. Cloos, M.; Sapiie, B.; Van Ufford, A.Q.; Weiland, R.J.; Warren, P.Q.; McMahon, T.P. Collisional Delamination in New Guinea: The Geotectonics of Subducting Slab Breakoff. Spec. Pap. Geol. Soc. Am. 2005, 400, 1-51. [CrossRef]

47. Hill, K.C.; Hall, R. Mesozoic-Cenozoic Evolution of Australia's New Guinea Margin in a West Pacific Context. In Special Paper of the Geological Society of America; Geological Society of America: Boulder, CO, USA, 2003; Volume 372, pp. 265-290. [CrossRef]

48. Dickinson, W.R. Forearc Basins. In Tectonics of Sedimentary Basins; Busby, C.J., Ingersoll, R.V., Eds.; Blackwell Science: Oxford, UK, 1995; pp. 221-262.

49. Arai, S. Chemistry of Chromian Spinel in Volcanic Rocks as a Potential Guide to Magma Chemistry. Mineral. Mag. 1992, 56, 173-184. [CrossRef]

50. Schulze, D.J. Origins of Chromian and Aluminous Spinel Macrocrysts from Kimberlites in Southern Africa. Can. Mineral. 2001, 39, 361-376. [CrossRef]

51. Deer, W.A.; Howie, R.A.; Zussman, J. Rock-Forming Minerals. In Volume 2A: Single-Chain Silicates; Geological Society of London: London, UK, 1997; Volume 2A, pp. 198-290. ISBN 1897799853.

52. Pubellier, M.; Ali, J.; Monnier, C. Cenozoic Plate Interaction of the Australia and Philippine Sea Plates: "Hit-and-Run" Tectonics. Tectonophysics 2003, 363, 181-199. [CrossRef]

53. Pubellier, M.; Monnier, C.; Maury, R.; Tamayo, R. Plate Kinematics, Origin and Tectonic Emplacement of Supra-Subduction Ophiolites in SE Asia. Tectonophysics 2004, 392, 9-36. [CrossRef]

54. Zhou, M.F.; Robinson, P.T.; Su, B.X.; Gao, J.F.; Li, J.W.; Yang, J.S.; Malpas, J. Compositions of Chromite, Associated Minerals and Parental Magmas of Podiform Chromite Deposits: The Role of Slab Contamination of Asthenospheric Melts in Suprasubduction Zone Environments. Gondwana Research. 2014, 26, 262-283. [CrossRef]

55. Dick, H.J.B.; Bullen, T. Chromian Spinel as a Petrogenetic Indicator In Abyssal and Alpine-Type Peridotites and Spatially Associated Lavas. Contrib. Mineral. Petrol. 1984, 86, 54-76. [CrossRef]

56. Morton, A.C.; Hallsworth, C.R. Processes Controlling the Composition of Heavy Mineral Assemblages in Sandstones. Sediment. Geol. 1999, 124, 3-29. [CrossRef]

57. Garzanti, E.; Andò, S. Chapter 20 Heavy Mineral Concentration in Modern Sands: Implications for Provenance Interpretation. In Developments in Sedimentology; Mange, M.A., Wright, D.T., Eds.; Elsevier Science: Amsterdam, The Netherlands, 2007; Volume 58, pp. 517-541. [CrossRef]

58. Komar, P.D. The Entrainment, Transport and Sorting of Heavy Minerals by Waves and Currents. In Developments in Sedimentology; Mange, M.A., Wright, D.T., Eds.; Elsevier Science: Amsterdam, The Netherlands, 2007; Volume 58, pp. 3-44. [CrossRef]

59. Sevastjanova, I.; Hall, R.; Alderton, D. A Detrital Heavy Mineral Viewpoint on Sediment Provenance and Tropical Weathering in SE Asia. Sediment. Geol. 2012, 280, 179-194. [CrossRef]

60. Milliman, J.D. Sediment Discharge to the Ocean from Small Mountainous Rivers: The New Guinea Example. Geo-Marine Lett. 1995, 15, 127-133. [CrossRef]

61. Zglinicki, K. The Geological-Mineralogical Characteristic of the Contemporary Coastal Marine Sediments of Jayapura Regency (Indonesian Part of New Guinea). Ph.D. Thesis, University of Warsaw, Warsaw, Poland, 2016; pp. 1-163. (In Polish).

62. Kamenetsky, V.S.; Crawford, A.J.; Meffre, S. Factors Controlling Chemistry of Magmatic Spinel: An Empirical Study of Associated Olivine, Cr-Spinel and Melt Inclusions from Primitive Rocks. J. Petrol. 2001, 42, 655-671. [CrossRef]

63. Pearce, J.A. Supra-Subduction Zone Ophiolites. The Search for Modern Analogue. In Ophiolite Concept and the Evolution of Geologic Thought; Dilek, Y., Newcomb, S., Eds.; Geological Society of America: Boulder, CO, USA, 2003; pp. 269-294. [CrossRef]

64. Zhou, M.F.; Robinson, P.T.; Malpas, J.; Edwards, S.J.; Qi, L. REE and PGE Geochemical Constraints on the Formation of Dunites in the Luobusa Ophiolite, Southern Tibet. J. Petrol. 2005, 46, 615-639. [CrossRef] 
65. Stevens, R.E. Composition of Some Chromites of the Western Hemisphere. Am. Mineral. 1944, $29,1-34$.

66. Jan, M.Q.; Windley, B.F. Chromian Spinel-Silicate Chemistry in Ultramafic Rocks of the Jijal Complex, Northwest Pakistan. J. Petrol. 1990, 31, 667-715. [CrossRef]

67. Pagé, P.; Bédard, J.H.; Schroetter, J.M.; Tremblay, A. Mantle Petrology and Mineralogy of the Thetford Mines Ophiolite Complex. Lithos 2008, 100, 255-292. [CrossRef]

68. Dickey, J.S. A Hypothesis of Origin for Podiform Chromite Deposits. Geochim. Cosmochim. Acta 1975, 39, 1061-1074. [CrossRef]

69. Leblanc, M.; Nicolas, A. Ophiolitic Chromitites. Int. Geol. Rev. 1992, 34, 653-686. [CrossRef]

70. Zhou, M.F.; Robinson, P.T.; Bai, W.J. Formation of Podiform Chromitites by Melt/Rock Interaction in the Upper Mantle. Miner. Depos. 1994, 29, 98-101. [CrossRef]

71. Zhou, M.F.; Malpas, J.; Robinson, P.T.; Sun, M.; Li, J.W. Crystallization of Podiform Chromitites from Silicate Magmas and the Formation of Nodular Textures. Resour. Geol. 2001, 51, 1-6. [CrossRef]

72. Gervilla, F.; Proenza, J.A.; Frei, R.; González-Jiménez, J.M.; Garrido, C.J.; Melgarejo, J.C.; Meibom, A.; Díaz-Martínez, R.; Lavaut, W. Distribution of Platinum-Group Elements and Os Isotopes in Chromite Ores from Mayarí-Baracoa Ophiolitic Belt (Eastern Cuba). Contrib. Mineral. Petrol. 2005, 150, 589-607. [CrossRef]

73. González-Jiménez, J.M.; Griffin, W.L.; Proenza, J.A.; Gervilla, F.; O’Reilly, S.Y.; Akbulut, M.; Pearson, N.J.; Arai, S. Corrigendum to 'Chromitites in Ophiolites: How, Where, When, Why? Part II. The Crystallisation of Chromitites'. Lithos 2014, 189, 140-158. [CrossRef]

74. González-Jiménez, J.M.; Proenza, J.A.; Gervilla, F.; Melgarejo, J.C.; Blanco-Moreno, J.A.; Ruiz-Sánchez, R.; Griffin, W.L. High-Cr and High-Al Chromitites from the Sagua de Tánamo District, Mayarí-Cristal Ophiolitic Massif (Eastern Cuba): Constraints on Their Origin from Mineralogy and Geochemistry of Chromian Spinel and Platinum-Group Elements. Lithos 2011, 125, 101-121. [CrossRef]

75. Pagé, P.; Barnes, S.J. Using Trace Elements in Chromites to Constrain the Origin of Podiform Chromitites in the Thetford Mines Ophiolite, Québec, Canada. Econ. Geol. 2009, 104, 997-1018. [CrossRef]

76. Arai, S. Compositional Variation of Olivine-Chromian Spinel in Mg-Rich Magmas as a Guide to Their Residual Spinel Peridotites. J. Volcanol. Geotherm. Res. 1994, 59, 303-310. [CrossRef]

77. Zhou, M.F.; Robinson, P.T.; Malpas, J.; Li, Z. Podiform Chromitites in the Luobusa Ophiolite (Southern Tibet): Implications for Melt-Rock Interaction and Chromite Segregation in the Upper Mantle. J. Petrol. 1996, 37, 3-21. [CrossRef]

78. Pearce, J.A.; Barker, P.F.; Edwards, S.J.; Parkinson, I.J.; Leat, P.T. Geochemistry and Tectonic Significance of Peridotites from the South Sandwich Arc-Basin System, South Atlantic. Contrib. Mineral. Petrol. 2000, 139, 36-53. [CrossRef]

79. Zaccarini, F.; Garuti, G.; Proenza, J.A.; Campos, L.; Thalhammer, O.A.R.; Aiglsperger, T.; Lewis, J.F. Chromite and Platinum Group Elements Mineralization in the Santa Elena Ultramafic Nappe (Costa Rica): Geodynamic Implications. Geol. Acta. 2011, 9, 407-423. [CrossRef]

80. Rollinson, H. The Geochemistry of Mantle Chromitites from the Northern Part of the Oman Ophiolite: Inferred Parental Melt Compositions. Contrib. Mineral. Petrol. 2008, 156, 273-288. [CrossRef]

81. Maurel, C.; Maurel, P. Etude Experimentale de La Distribution de l'aluminium Entre Bain Silicate Basique et Spinelle Chromifere. Implications Petrogenetiques: Teneur En Chrome Des Spinelles. Bull. Mineral. 1982, 105, 197-202. [CrossRef]

82. Dönmez, C.; Keskin, S.; Günay, K.; Çolakoğlu, A.O.; Çiftçi, Y.; Uysal, İ; Türkel, A.; Yıldırım, N. Chromite and PGE geochemistry of the Elekdağ Ophiolite (Kastamonu, Northern Turkey): Implications for deep magmatic processes in a supra-subduction zone setting. Ore Geol. Rev. 2014, 57, 216-228. [CrossRef]

83. Hall, R. The Plate Tectonics of Cenozoic SE Asia and the Distribution of Land and Sea. In Biogeogr. Geol. Evol. SE Asia; Hall, R., Holloway, J.D., Eds.; Backhuys Publishers: Leiden, The Netherlands, 1998; pp. 99-131.

(C) 2020 by the authors. Licensee MDPI, Basel, Switzerland. This article is an open access article distributed under the terms and conditions of the Creative Commons Attribution (CC BY) license (http://creativecommons.org/licenses/by/4.0/). 